\title{
SLUG-STOCHASTICALLY LIGHTING UP GALAXIES. I. METHODS AND VALIDATING TESTS
}

\author{
Robert L. Da Silva ${ }^{1}$, Michele Fumagalli, and Mark Krumholz \\ Department of Astronomy and Astrophysics, UCO/Lick Observatory, University of California, 1156 High Street, Santa Cruz, CA 95064, USA \\ Received 2011 June 6; accepted 2011 October 18; published 2012 January 12
}

\begin{abstract}
The effects of stochasticity on the luminosities of stellar populations are an often neglected but crucial element for understanding populations in the low-mass or the low star formation rate regime. To address this issue, we present SLUG, a new code to "Stochastically Light Up Galaxies." SLUG synthesizes stellar populations using a Monte Carlo technique that properly treats stochastic sampling including the effects of clustering, the stellar initial mass function, star formation history, stellar evolution, and cluster disruption. This code produces many useful outputs, including (1) catalogs of star clusters and their properties such as their stellar initial mass distributions and their photometric properties in a variety of filters, (2) two dimensional histograms of color-magnitude diagrams of every star in the simulation, and (3) the photometric properties of field stars and the integrated photometry of the entire simulated galaxy. After presenting the SLUG algorithm in detail, we validate the code through comparisons with STARBURST99 in the well-sampled regime, and with observed photometry of Milky Way clusters. Finally, we demonstrate SLUG's capabilities by presenting outputs in the stochastic regime. SLUG is publicly distributed through the Web site http://sites.google.com/site/runslug/.
\end{abstract}

Key words: galaxies: star clusters: general - galaxies: stellar content - methods: numerical - methods: statistical stars: formation - techniques: photometric

Online-only material: color figures

\section{INTRODUCTION}

Fundamental progress in understanding the properties of galaxies, star clusters, and stellar populations comes from the comparisons between the observed and synthetic photometry derived from stellar evolution codes. It has become common practice to infer properties such as star formation rate (SFR), star formation history (SFH), age, metallicity, redshift, and stellar mass from photometry. Despite the limits of theoretical modeling of stellar populations (such as uncertainties with dust, stellar evolution, and the stellar initial mass function (IMF); see Conroy et al. 2009, 2010; Conroy \& Gunn 2010), synthetic libraries have reached a degree of precision that allows accurate estimates of these parameters-although sometimes with degeneracy-in massive galaxies and clusters.

However, observations reveal a higher complexity in lower mass systems where scaling relations which apply to more massive systems cannot be trivially extrapolated (e.g., Lee et al. 2007; Weisz et al. 2008). Moreover, in lower mass systems, the limited number of stars that are present render these systems inconsistent with the predictions of most of the currently available codes for synthetic photometry (such as STARBURST99 (SB99), Leitherer et al. 1999; PEGASE, Fioc \& Rocca-Volmerange 1997; or GALEV, Kotulla et al. 2009). This is because these stellar population models predict only the mean luminosities in given bands, assuming many realizations of the populations. In reality, the individual realizations may have significant scatter about these mean luminosities. For this reason, it is safe to compare them to individual observations only when the coeval stellar populations being observed are quite large. Violation of this last condition leads to stochastic variations in the photometric properties, but these codes are not designed to fully capture them.

\footnotetext{
1 NSF Graduate Research Fellow.
}

For example, in globular clusters, some of the simplest observed stellar populations, failure to account for sampling effects can lead to a significant error in the estimated contributions of blue horizontal-branch and asymptotic giant branch (AGB) stars to the integrated light. As a result, correct estimates of globular cluster ages and metallicities based on their integrated light are possible only if one correctly accounts for this stochasticity (Brocato et al. 1999; Colucci et al. 2011).

Moreover, in weakly star-forming regions, stochastic effects can mimic those of a varying IMF. Indeed, recent observations in the low-SFR regime have led to serious consideration of a varying IMF (Pflamm-Altenburg \& Kroupa 2008; Hoversten \& Glazebrook 2008; Meurer et al. 2009; Lee et al. 2009). However, a fully self-consistent model of stochasticity, allowing for a range of parameters such as differing degrees of stellar clustering, metallicities, stellar tracks, input IMFs and initial cluster mass functions (ICMFs), and SFHs, has not been available to test the null hypothesis of a non-varying but stochastically sampled IMF.

These considerations apply not only to the dwarf galaxies studied by Lee et al. (2009) but also to the outer regions of galaxies such as XUV disks (Boissier et al. 2007; Thilker et al. 2007) and outlying H II regions (Werk et al. 2008; Gogarten et al. 2009) where the stochasticity becomes crucial in the interpretation of inferred SFRs and SFHs.

While the number of studies that use Monte Carlo approaches to address problems on scales of clusters and galaxies is growing (e.g., Raimondo et al. 2005; Popescu \& Hanson 2009; SilvaVilla \& Larsen 2011; Fouesneau \& Lançon 2010; Eldridge 2011), a general-purpose tool to study photometry in clusters and galaxies has not previously been available. To fill this need, we have created SLUG, a code that allows proper study of the stochastic star formation regime at a range of scales from individual star clusters to entire galaxies. SLUG provides a variety of tools for studying the stochastic regime, such as the ability to create catalogs of clusters including their individual IMFs and 
photometric properties, and color-magnitude diagrams (CMDs) of entire galaxies where we keep track of the photometry of every star as well as integrated photometry of entire composite stellar populations.

This paper, the first of a series, focuses on the methods used in the code along with several tests to demonstrate that we are reliably reproducing observations and predictions from other codes for synthetic photometry. We then demonstrate the use of this code in the stochastic regime. In a companion paper (Fumagalli et al. 2011a), we use SLUG to show that, once random sampling is included, a stochastic non-varying IMF can reproduce the observed variation of the $\mathrm{H} \alpha /$ far-UV (FUV) ratio in dwarf galaxies, without resorting to modifications of the IMF. In the second paper of the series (R. L. da Silva et al. 2011 , in preparation) we will explore in detail the implications of stochastic star formation with clustering. Further work will apply this code to a variety of astrophysical questions, such as understanding SFR calibrations in the stochastic regime and further study of other claims of IMF variation.

The layout of the paper is as follows: Section 2 presents an introduction to stochasticity and its effects on the luminosity of stellar populations; Section 3 gives a detailed description of the SLUG algorithm; Section 4 discusses various tests of the code; Section 5 shows a presentation of the code's outputs in the stochastic regime; and finally, Section 6 summarizes the results.

\section{WHAT IS STOCHASTICITY?}

Many astrophysical studies require the creation of synthetic photometry of galaxies and other collections of stars in order to compare with observations. In this section we present a discussion of the various effects of stochasticity and the regimes in which they are important.

\subsection{Coeval Stellar Populations}

The standard procedure for calculating the luminosity from a coeval population of stars used by the most popular implementations (such as SB99) is as follows (Tinsley 1980; Scalo 1986).

To find the mean luminosity of a coeval population of stars with initial mass $M$ (i.e., mass at birth, before any mass loss due to stellar evolution) in some band $\beta$ at a time $t$ after formation $\left(L_{\beta \text {,coeval }}(t)\right)$, one simply integrates the luminosity of each star in that band as a function of the initial stellar mass $m$ and time $\left(L_{\beta}(m, t)\right)$, weighting by the distribution of initial stellar masses (i.e., the IMF) $d N / d m$ :

$$
L_{\beta, \text { coeval }}(t)=\left(\frac{M}{M_{\odot}}\right) \int_{m_{\min }}^{m_{\max }} L_{\beta}(m, t) \frac{d N}{d m} d m,
$$

where $m_{\min }$ and $m_{\max }$ are the minimum and maximum initial stellar masses allowed by the IMF, and we normalize the IMF such that $\int m(d N / d m) d m=1 M_{\odot}$. Note that the mean total luminosity simply scales linearly with the initial mass of the stellar population. However, for small stellar populations, any individual set of stars drawn from the given mass distribution may have a luminosity that deviates significantly from the mean. This is because each realization of a given mass $M$ is built up with a different sampling of stellar masses which, due to the nonlinear dependence of luminosity on stellar mass, yields a different luminosity. For example, if one realization of a stellar population with a total mass of $20 M_{\odot}$ consists of one $20 M_{\odot}$ star, its total luminosity will be quite different than if the same population were composed of twenty $1 M_{\odot}$ stars. We call this type of stochastic process sampling stochasticity. When stochastic sampling is important, the distribution of luminosities can be both very broad and highly asymmetric, and so it becomes important to know the shape of the luminosity distribution as well as its mean.

One of the more significant manifestations of sampling stochasticity is the apparent undersampling of the upper end of the IMF. Since the IMF is steeply declining with increasing stellar mass, it is improbable that a low-mass population will contain a massive star. As a result, the IMF in a low-mass population with few stars can often appear truncated and less luminous than a fully sampled assumption would have predicted. ${ }^{2}$ When considering young clusters, those that are not well populated at the upper end of the IMF can appear much less luminous since the luminosity dependence on mass is much steeper than the slope of the IMF, resulting in the majority of the light being produced by the most massive stars.

One can roughly estimate the mass below which this effect is significant by calculating the expectation value of obtaining a star above a given mass. We do so following the formalism of Elmegreen (2000), who find that the total mass $(M)$ required to expect a single star above a mass $m$ is

$$
M \sim 3 \times 10^{3}\left(\frac{m}{100 M_{\odot}}\right)^{1.35} .
$$

This statement is clearly dependent on one's choice of IMF. Elmegreen (2000) uses a Salpeter IMF with a lower limit of $0.3 M_{\odot}$ and no upper limit. (However, if one imposes an upper limit as done by Weidner \& Kroupa 2004, the result does not change significantly.) This result implies that in order to reasonably expect even a single $120 M_{\odot}$ star, $^{3}$ one would need to sample approximately $10^{4} M_{\odot} \equiv M_{\text {trunc }}$. Thus, one can ignore this apparent pseudo truncation and other sampling effects only for coeval populations with masses $\gg M_{\text {trunc }}$. (Additional reference on the limits of stochastic sampling including stellar evolution can be found in Cerviño \& Luridiana 2004, 2006. For specific considerations to $\mathrm{H} \alpha$ luminosity, one of the features of a stellar population most sensitive to stochasticity, see Cerviño et al. 2003.)

Another manner in which stochastic sampling can manifest in coeval populations is for stars going through particularly short-lived and luminous phases of evolution after they leave the main sequence (e.g., AGB and blue horizontal-branch stars; Buzzoni 1989; Lançon \& Mouhcine 2000; Lançon 2010; Fouesneau \& Lançon 2010). Since these phases are short, only a very narrow range of masses is undergoing one of them at any given time. Thus, the exact sampling within that mass range can have a significant impact on the number of stars in that phase. As a result, a non-infinite population of stars can have additional random scatter in luminosity even if $M>M_{\text {trunc }}$. This effect is more important in populations with little ongoing star formation relative to their stellar mass (otherwise new stars dominate the photometric properties of the population), at specific ages when these post-main-sequence populations contribute significantly to the luminosity of the population (Colucci et al. 2011).

\footnotetext{
2 Extremely rare drawings of the IMF at low masses can also produce pseudo top-heavy IMFs which are overly luminous per unit mass.

3 Due to limitations of stellar evolutionary tracks, this is the highest stellar mass SLUG can model and is a reasonable guess for the highly uncertain absolute stellar mass limit. While some (e.g., Figer 2005) suggest a value of $\sim 120-150 M_{\odot}$, others (e.g., Crowther et al. 2010) suggest it may be as high as $300 M_{\odot}$.
} 


\subsection{Composite Stellar Populations}

In order to characterize a more complicated $\mathrm{SFH}$, the next step is to integrate over the coeval populations discussed above to find the luminosity of all stars in a given band at a time $\tau$,

$$
L_{\beta, \text { total }}(\tau)=\int_{-\infty}^{\tau} \frac{\operatorname{SFR}(t)}{M_{\odot}} L_{\beta, \text { coeval }}(\tau-t) d t,
$$

where $\operatorname{SFR}(t)$ is the SFR as a function of time.

In order to treat such models as representative, two conditions must be met: (1) each of the summed coeval populations is large enough to ignore the effects of sampling stochasticity and (2) the SFR is continuously sampled as well. These conditions can quickly break down for sufficiently low SFRs.

To illustrate this point, consider a galaxy forming stars at a constant rate. In order for stochastic sampling effects to be negligible within some time interval $d t$, there need to be at least $M_{\text {trunc }}$ worth of stars formed in that interval. For the SFR to be considered reasonably well sampled, $d t$ must be much smaller than the evolutionary timescales of any of the stars, which are $\approx 10^{6} \mathrm{yr}$ for the massive stars that generally dominate the light in an actively star-forming system. Thus, the condition for stochastic sampling effects to be negligible is that

$$
d t=\frac{M_{\text {trunc }}}{\mathrm{SFR}} \ll 10^{6} \mathrm{yr},
$$

implying that this condition is met only for SFRs consistently $\gg 10^{-2} M_{\odot} \mathrm{yr}^{-1} \equiv \mathrm{SFR}_{\mathrm{temp}}$. However, this temporal stochasticity is amplified when one considers that stars are believed to be formed in discrete collections known as clusters. As a result, the clumping in time of star formation in clusters can produce stochastic effects even in regions with SFRs higher than SFR temp. In this case the characteristic mass in Equation (4) is replaced with a mass characteristic of the clusters being drawn (discussed further in R. L. da Silva 2011, in preparation; Fumagalli et al. 2011a).

The conditions required to ignore the effects of stochasticity break down in a variety of astrophysical environments such as dwarf galaxies (e.g., Lee et al. 2009), low SFR regions in the outskirts of galaxies (e.g., Boissier et al. 2007; Fumagalli \& Gavazzi 2008; Bigiel et al. 2010), and low surface brightness galaxies (e.g., Boissier et al. 2008).

\section{TECHNIQUE}

\subsection{Overview}

Here we present a brief overview of the code while we present each step in detail in the subsequent sections.

SLUG simulates star formation according to the scheme presented in Figure 1. We create collections of star clusters obeying a user-defined ICMF (which can include a given mass fraction of stars not formed in clusters), SFH, IMF, and choice of stellar evolutionary tracks. This collection defines a "galaxy." We do not currently include any effects of chemical evolution. A description of the parameters that users can vary is provided in Table 1 and will be described in the following sections.

These galaxies are built up (Section 3.2) by first drawing the age of the cluster from a distribution defined by the given $\mathrm{SFH}$. The mass of the cluster is drawn from the ICMF. Next, the cluster's mass is then filled up with stars according to an IMF. Each of the stars within the cluster is evolved using a stellar evolutionary track combined with a model spectral
Table 1

Input Parameters

\begin{tabular}{ll}
\hline \hline Parameter & \multicolumn{1}{c}{ Description } \\
\hline IMF & Controlling the Physics \\
& $\begin{array}{l}\text { Krollar initial mass function; can choose } \\
\text { an arbitrary slope }\end{array}$ \\
ICMF & $\begin{array}{l}\text { Initial cluster mass function; can change } \\
\text { slope, minimum and maximum mass }\end{array}$ \\
Stellar evolutionary tracks & Library of models used for stellar evolution \\
Metallicity & Metallicity of the stellar population \\
Stellar atmosphere & Which scheme and models are used for SEDs \\
Stellar wind model & Which wind model is used for SEDs \\
Fraction of stars in clusters & Mass fraction of stars formed in clusters \\
\hline & Controlling the Simulation \\
\hline Maximum time & How long the simulation is run \\
SFH & Can be arbitrary \\
Seed & Random seed used for simulation \\
\hline & Controlling Output \\
\hline Time step & Time between code outputs \\
Fluxes & Choose which fluxes to output \\
Colors & Which colors to use for CMDs \\
CMD output parameters & Choice of number of bins and \\
range of color and luminosity for each CMD & Set to print output for each cluster \\
& Set to output IMF histograms for each cluster \\
&
\end{tabular}

Note. ${ }^{\text {a }}$ Only applicable for Schmutz (1998) O star atmospheres in Lejeune+Sch models (see Table 3).

energy distribution (SED) to determine a variety of integrated fluxes corresponding to commonly used photometric filters (Section 3.3).

At a given set of time steps, these fluxes are summed over each star cluster. The clusters are then disrupted according to the prescription of Fall et al. (2009). Disrupted clusters have their fluxes added to a "field" population while surviving clusters have their properties stored individually.

The code repeats these operations until a stellar mass equal to the integral of the provided SFH is created. The run time of the code is roughly

$$
\begin{aligned}
t_{\text {run }} & \sim 4 \mathrm{~s}+(60 \mathrm{~s})\left(1+6.6\left[1-f_{c}\right]\right)\left(\frac{\int_{0}^{t_{\max }} \operatorname{SFR}(t) d t}{10^{7} M_{\odot}}\right) \\
& \times\left(\frac{\text { time steps }}{5}\right)\left(\frac{\text { No. filters }}{2}\right)\left(\frac{\text { cpu Speed }}{2.33 \mathrm{GHz}}\right),
\end{aligned}
$$

where $f_{c}$ is the fraction of stars in clusters and $t_{\max }$ is the maximum run time of the simulation.

The code outputs a variety of files that keep track of the properties of the stars, clusters, and total integrated stellar populations. Table 2 provides a short description of each available output file. All outputs are parsed and transformed into binary FITS tables.

The code is open source and written in $\mathrm{C}++$ with wrapping and parsing routines written in IDL. This entire process can be controlled through an IDL graphical user interface (see Figure 2) or either the UNIX or IDL command lines. The IDL routines are also available wrapped in packages for use with the IDL virtual machine ${ }^{4}$ for those without IDL licenses. SLUG is available for

\footnotetext{
4 The IDL virtual machine is freely available from http://www.ittvis.com/language/en-us/productsservices/ idl/idlmodules/idlvirtualmachine.aspx.
} 


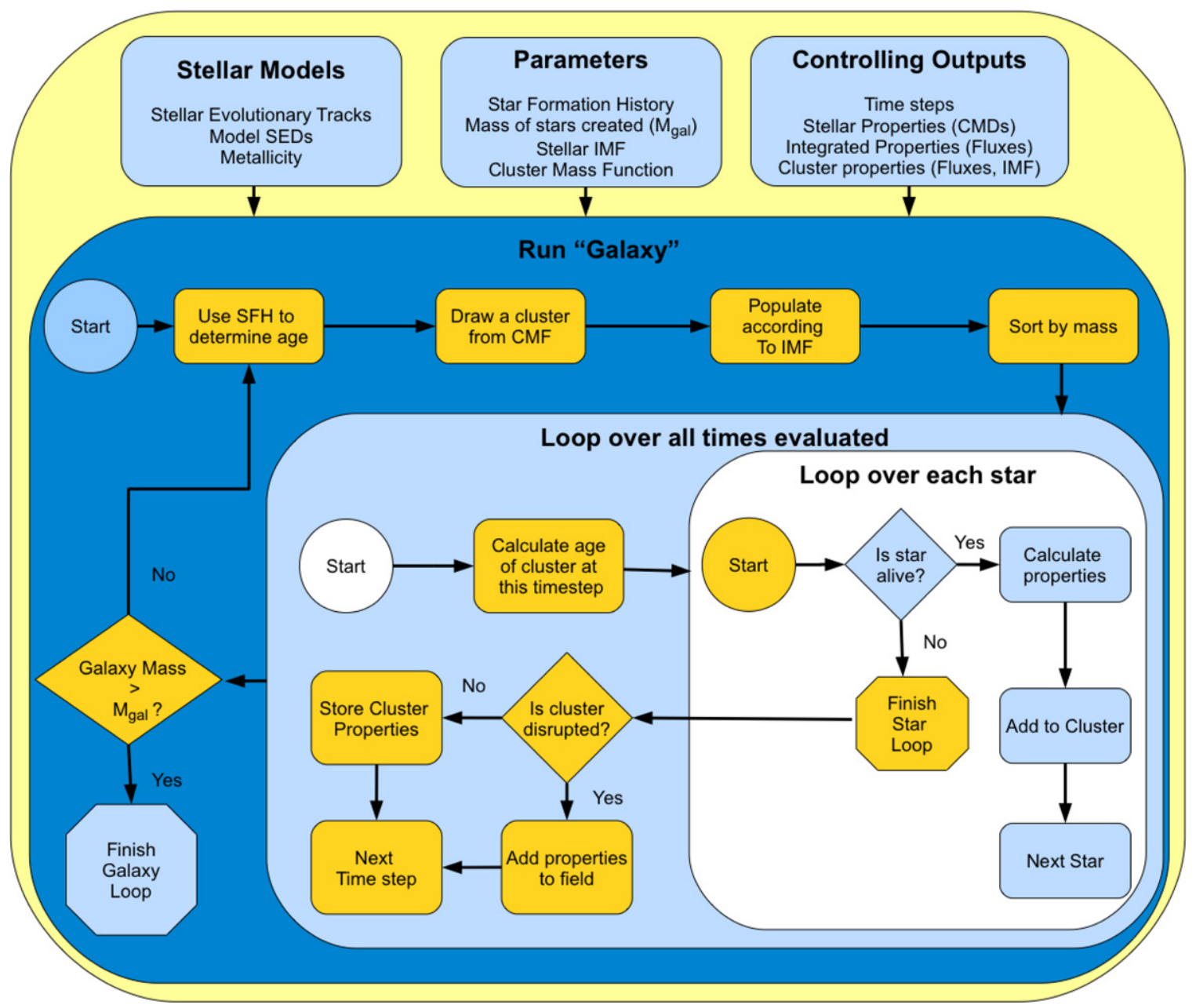

Figure 1. Schematic flowchart describing the algorithm of the SLUG code. Note that for the case of unclustered star formation, the cluster mass is drawn from the IMF and the population step is skipped as the single star is treated as part of a disrupted cluster for the remainder of the code. Note this is updated from Fumagalli et al. (2011b).

(A color version of this figure is available in the online journal.)

Table 2

SLUG Output Files

\begin{tabular}{ll}
\hline \hline Name & \multicolumn{1}{c}{ Description } \\
\hline Histogram & $\begin{array}{l}\text { A two-dimensional histogram of the user's choice of color-magnitude diagram(s) } \\
\text { of every star in the "galaxy" at each time step } \\
\text { Mass, fluxes of most massive star born in cluster, number of stars formed in cluster, and } \\
\text { age of each undisrupted cluster at each time step }\end{array}$ \\
Cluster & $\begin{array}{l}\text { A histogram of the IMF of each cluster that appears in the Cluster file } \\
\text { The total flux of the entire "galaxy" at each time step }\end{array}$ \\
Integral & $\begin{array}{l}\text { The total stellar mass actually formed, } \\
\text { as well as the actual SFH and ICMF of the simulation }\end{array}$ \\
\end{tabular}

download with an up-to-date manual; visit the SLUG Web site at http://sites.google.com/site/runslug/.

\subsection{Cluster Creation}

Most stars are thought to be born in star clusters (Lada \& Lada 2003) and the distribution of star cluster masses appears to obey a power-law distribution, where observations (e.g., Zhang \& Fall 1999; Lada \& Lada 2003; Fall et al. 2009; Chandar et al. 2010) and theory (e.g., Fall et al. 2010) suggest that the index $(\beta)$ of the power law $d N / d M \propto M^{-\beta}$ is approximately 2 . SLUG allows for both clustered and unclustered star formation. The user can choose what fraction of the stellar mass is formed in star clusters. If the code is forming clusters $\left(f_{c}>0\right)$, the ICMF's power-law slope as well as its upper and lower bounds can be varied. If unclustered star formation is desired $\left(f_{c}=0\right)$, the stars' masses are drawn individually from an IMF and treated as a disrupted "cluster" of one star for the remainder of the code.

The initial masses of stars are drawn from an IMF. Choices of IMF ${ }^{5}$ currently are Chabrier (2003), Kroupa (2001), Salpeter (1955), a user-defined arbitrary power law, and the recently proposed IGIMF (Kroupa \& Weidner 2003; Pflamm-Altenburg

\footnotetext{
5 IMFs are truncated to $0.08-120 M_{\odot}$ due to the lack of stellar tracks outside
} that range. 


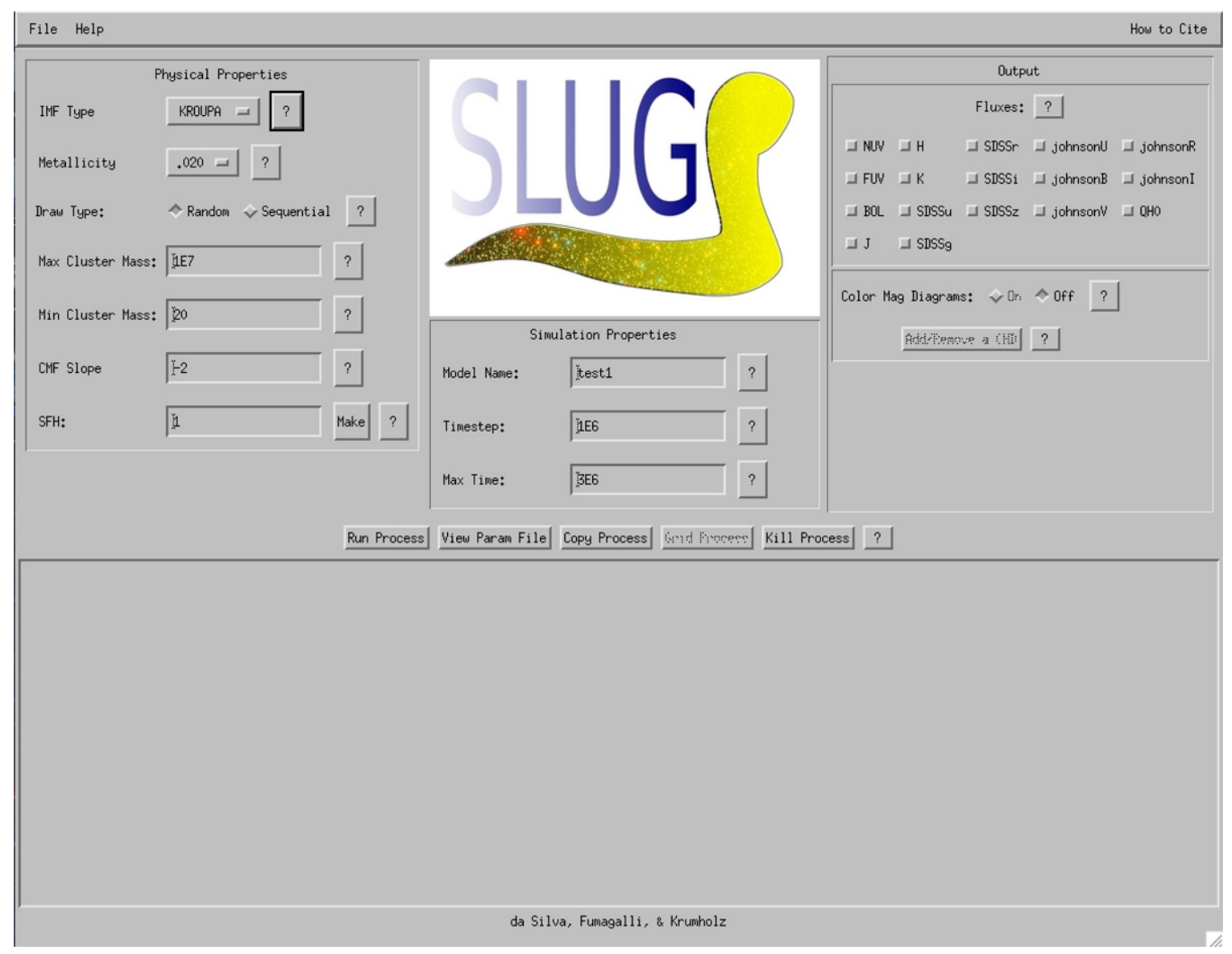

Figure 2. IDL GUI interface for running the code. The code may also be called via the UNIX or IDL command lines.

(A color version of this figure is available in the online journal.)

\& Kroupa 2008). While the Chabrier, Kroupa, Salpeter, and power-law IMFs are implemented as a standard probability density function of stellar masses, the IGIMF has additional features that require different treatment (see Appendix A).

Regardless of the choice of IMF, we draw stars until the total mass of the star cluster is built up. Since the random distribution of stars never exactly equals the mass of the cluster, a question arises as to whether to keep the last star added. This last star increases the mass of the cluster above the cluster mass drawn from the ICMF. We determine whether or not to keep that star in the cluster based on whether keeping the star in makes the total mass of stars closer to the mass drawn from the ICMF than leaving it out. ${ }^{6}$

Independent of its mass, the age of the cluster relative to the galaxy is assigned in a probabilistic manner weighted by the SFH (which can be arbitrary) such that the SFH is reproduced on average. This is analogous to how the full IMF is reproduced on average when one combines many realizations of clusters, but individual realizations can be top-heavy or appear truncated. Note that this method produces a scatter in the SFHs for even a given "constant" SFR. Thus, SLUG's definition of a galaxy with a constant SFR is not a galaxy where the SFR is instantaneously constant at all times, ${ }^{7}$ but rather a galaxy that produces a mass

\footnotetext{
6 The effects of different sampling methods and their dependence on the ICMF is studied in detail by Haas \& Anders (2010). Our method is identical to their "stop-nearest" method.

7 A constant SFR cannot be instantaneously constant because stars form in discrete units of mass. For example, when a star is born, the instantaneous SFR is infinite. Thus, we must turn to a more probabilistic interpretation of the SFR.
}

of stars over a time $d t$ equal to $\mathrm{SFR} \times d t$, which is distributed in clusters whose ages are drawn from a uniform distribution. This interpretation of what an SFR is and its implications is discussed in more detail in R. L. da Silva et al. (2011, in preparation).

Clusters are born until the total mass of stars formed is equal to the integral of the SFH. As with the problem of populating a cluster with stars, a galaxy will never be filled to exactly its given mass with an integer number of clusters. Therefore, we apply the same condition for populating the galaxy as we do for the clusters: we add clusters until we exceed the galaxy mass (defined as the integral of the SFH) and keep the final cluster only if the updated total galaxy mass is closer to the desired value. As a result the average SFR over the entire simulation of a particular galaxy can be higher or lower than the input value. This effect is small for most regimes, but very rare drawings of the ICMF at low SFRs can produce mild departures. We emphasize that this is not the effect of any error associated with the code but rather is the necessary result of our interpretation of what an SFR means. This behavior is analogous to the situation that arises when drawing from the IMF: the total mass of stars drawn will never exactly match the target cluster mass, and for rare drawings it is possible that the actual cluster mass will differ from the target mass by a non-trivial fraction.

We demonstrate the results of this procedure in Figure 3. While lower average SFRs tend to produce larger fractional scatter in the instantaneous SFR, significant scatter remains until the SFRs exceed $10 M_{\odot} \mathrm{yr}^{-1}$. This scatter is a direct result of the finite size of clusters. This type of "bursty" behavior may be responsible for the observed bursty SFHs of dwarf galaxies 


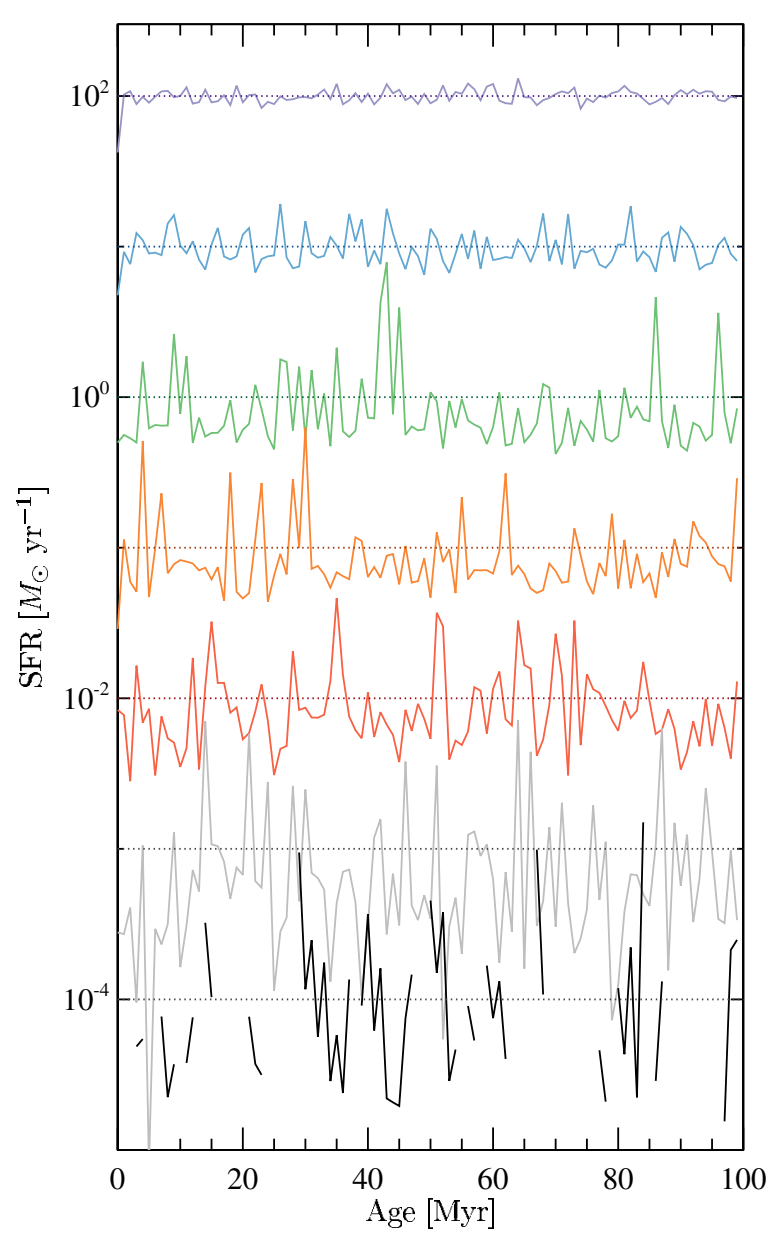

Figure 3. Examples of star formation histories averaged over $1 \mathrm{Myr}$ bins for simulations with varying input constant SFRs of $0.0001-100 M_{\odot} \mathrm{yr}^{-1}$. The dotted lines show the input SFR. The average SFR of the simulation in each case is within $2,0.2$, and $<0.02$ percent of the input for $10^{-4}, 10^{-3}$, and $>10^{-2} M_{\odot} \mathrm{yr}^{-1}$, respectively. SFRs of zero are masked.

(A color version of this figure is available in the online journal.)

(Weisz et al. 2011). To clarify with an example, consider that a $10^{7} M_{\odot}$ cluster (when averaged over 1 Myr similarly to the curves shown in Figure 3) will appear as a deviant peak for all but the highest SFRs, where the contribution of that individual cluster is drowned out by enough other clusters. Of course, averaging over a larger time interval $(\delta t)$ reduces the influence of any single cluster, and in the limit of averaging over arbitrarily large intervals the difference between the average and input SFR $\left(\Delta_{\text {avg-input }}\right)$ must approach zero. The rate at which $\Delta_{\text {avg-input }}$ decreases as a function of $\delta t$ is a function of the ICMF.

We note that in this release of the code all stars in a cluster are treated as having identically the same age, which may not be the case (e.g., see Bernasconi \& Maeder 1996). While observations suggest a scatter of a several Myr (Palla \& Stahler 1999; Jeffries 2007; Hosokawa et al. 2011), the mass dependence of this scatter is unclear. Given these uncertainties, and that the intracluster age scatter is at most a few Myr (typically small compared to the cluster age distribution), we chose to neglect this effect for now.

\subsubsection{Example of Cluster Creation Algorithm}

To illustrate our procedure for forming stars in clusters, we now give an example. Suppose a user requests an SFH consisting of a constant SFR of $2 M_{\odot} \mathrm{yr}^{-1}$ for $10^{6} \mathrm{yr}$, with an ICMF restricted to the mass range $10^{5}-10^{7} M_{\odot}$. In response, the
Table 3

Stellar Properties

\begin{tabular}{|c|c|}
\hline Parameter & Allowed Values \\
\hline Tracks & Geneva STD ${ }^{\mathrm{a}}$, Geneva $\mathrm{High}^{\mathrm{b}}$, Padova STD ${ }^{\mathrm{c}}$, Padova AGB $^{\mathrm{d}}$ \\
\hline \multirow[t]{2}{*}{ Metallicity $^{\mathrm{e}}$} & Geneva: $0.001,0.004,0.008,0.020,0.040$ \\
\hline & Padova: $0.0004,0.004,0.008,0.020,0.50$ \\
\hline SEDs & Planck $^{\mathrm{f}}$, Lejeune $^{\mathrm{g}}$, Lejeune+Sch ${ }^{\mathrm{h}}$, Lejeune $+\mathrm{Smi}^{\mathrm{i}}, \mathrm{Pau}+\mathrm{Smi}$ \\
\hline Wind Models ${ }^{\mathrm{k}}$ & Maeder $^{\mathrm{l}}$, Empirical $^{\mathrm{m}}$, Theoretical $^{\mathrm{m}}$, Elson $^{\mathrm{n}}$ \\
\hline
\end{tabular}

Notes.

a Charbonnel et al. (1996, 1999).

b Same as "a," but for high-mass stars use higher mass loss rate models from Meynet et al. (1994).

${ }^{c}$ Fagotto et al. (1994a, 1994b) and references therein.

d Same as "c" except use Padova+AGB implementation from Vázquez \& Leitherer (2005).

e Solar is 0.20 .

${ }^{f}$ Simple blackbody SED.

g Lejeune et al. (1997, 1998).

h Same as "g," but for O stars use Schmutz (1998).

i Same as "g," but for O stars use the Smith et al. (2002) implementation of the Hillier \& Miller (1998).

j Same as "i," but also include the Smith et al. (2002) implementation of Pauldrach et al. (2001) atmospheres.

k Only relevant when using Schmutz (1998) atmospheres with Lejeune+Sch models.

${ }^{1}$ de Jager et al. (1988); Maeder \& Meynet (1987); Maeder (1990).

${ }^{\mathrm{m}}$ Leitherer et al. (1992).

${ }^{n}$ Elson et al. (1989).

code starts by determining an age for the first cluster. This age is drawn from a distribution that is exactly equal to the normalized SFH. In this example, the SFR is constant so the $\mathrm{SFH}$ is flat and hence the distribution from which the age is drawn is simply a uniform probability from 0 to $10^{6} \mathrm{yr}$. Suppose the code draws an age of $5 \times 10^{5} \mathrm{yr}$ for the first cluster. Once the age has been determined, the code then draws a cluster mass from the ICMF. Suppose this mass turns out to be $1.6 \times 10^{6} M_{\odot}$. The code then populates that cluster with stars until the total mass of stars is greater than $1.6 \times 10^{6} M_{\odot}$. Since the total mass of stars formed at this point does not exceed the integral of the SFH $\left(\int_{0}^{10^{6} \mathrm{yr}} 2 M_{\odot} \mathrm{yr}^{-1} d t=2 \times 10^{6} M_{\odot}\right)$, the code draws another cluster. Suppose that this time the draw results in a cluster age of $1 \times 10^{5} \mathrm{yr}$ and a cluster mass of $5 \times 10^{5} M_{\odot}$. At this point the code has created a total mass in clusters that is greater than the integral of the SFR $\left(1.6 \times 10^{6} M_{\odot}+5 \times 10^{5} M_{\odot}=2.1 \times 10^{6} M_{\odot}>2 \times 10^{6} M_{\odot}\right)$. Because the total stellar mass if the code keeps the last cluster $\left(2.1 \times 10^{6} M_{\odot}\right)$ is closer to the integral of the SFR $\left(2 \times 10^{6} M_{\odot}\right)$ than if it discards the last cluster $\left(1.6 \times 10^{6} M_{\odot}\right)$, the code keeps the last cluster. It then fills that cluster from the IMF. At this point the code terminates, having drawn two clusters of mass $1.6 \times 10^{6} M_{\odot}$ and $5 \times 10^{5} M_{\odot}$ and ages of $5 \times 10^{5} \mathrm{yr}$ and $1 \times 10^{5} \mathrm{yr}$.

\subsection{Stellar Tracks, SEDs, and Broadband Photometry}

Given the mass and age of each star, we need to determine its properties for a variety of observables. We use the same algorithms adopted by SB99 (Leitherer et al. 1999; Vázquez \& Leitherer 2005) to create a set of tables over which SLUG interpolates the stellar photometry. These tables are constructed in advance to reduce the run time. The available tracks and SEDs are listed in Table 3. 
The first step in SLUG is to determine the physical properties of each star. To this end, we make use of a variety of stellar evolutionary models. Modifying the SB99 source code, we were able to obtain the full range of stellar tracks available to SB99 (Padova and Geneva; see Table 3). In the future we plan to implement a wider range of stellar tracks including those from Eldridge \& Stanway (2009) and the BaSTI library (Pietrinferni et al. 2007; Cordier et al. 2007). We supplement the Geneva tracks with the Padova+AGB tracks for stars in the mass range $0.15-0.8 M_{\odot}$. These models provide luminosities, gravities, chemical compositions, and effective temperatures at discrete intervals in the evolution of a discrete number of stellar masses. We then need to map these physical properties to stellar atmospheres in order to estimate the spectral energy distributions of the stars. SLUG allows users to choose from one of five possible SB99 algorithms for modeling the atmospheres. One possible model makes use of the Schmutz (1998) atmospheres which are dependent on the stellar wind model. For these models we implement all four prescriptions of stellar winds available in SB99 (see Table 3). It is important to note that the SB99 algorithms match SEDs to tracks with a nearest neighbor approach and not through interpolation. Therefore, there can be some mild discreteness in the output SEDs. Future work will include removal of this effect.

With SEDs in hand, we can convolve with filters to determine the photometry of each point in our stellar tracks. For this step we include the effects of nebular continuum (free-free, free-bound, and two photon processes) as implemented in SB99, but neglect nebular line emission for this first release of the code. (For a discussion of the importance of nebular continuum for the SEDs, see Reines et al. 2010, Leitherer \& Heckman 1995, and Mollá et al. 2009.) The full list of available filters is presented in Table 4. We also integrate the SED to determine the bolometric luminosity as well as to calculate $\mathrm{Q}\left(\mathrm{H}^{0}\right)$, the number of hydrogen ionizing photons emitted per second. One can convert $\mathrm{Q}\left(\mathrm{H}^{0}\right)$ to $\mathrm{H} \alpha$ luminosity with a simple conversion assuming case $\mathrm{B}$ recombination. Following the notation of Osterbrock \& Ferland 2006 ,

$$
\begin{aligned}
L_{\mathrm{H} \alpha} & =\left(1-f_{\mathrm{esc}}\right)\left(1-f_{\text {dust }}\right) \mathrm{Q}\left(\mathrm{H}^{0}\right)\left(\frac{\alpha_{\mathrm{H} \alpha}^{\mathrm{eff}}}{\alpha_{B}}\right) h \nu_{\mathrm{H} \alpha} \\
& \approx 1.37 \times 10^{-12}\left(1-f_{\mathrm{esc}}\right)\left(1-f_{\text {dust }}\right) \mathrm{Q}\left(\mathrm{H}^{0}\right) \operatorname{ergs~s}^{-1},
\end{aligned}
$$

where $f_{\text {esc }}$ is the escape fraction (poorly constrained but thought to be between 0.05 (Boselli et al. 2009) and 0.4 (Hirashita et al. 2003)) and $f_{\text {dust }}$ represents the fraction of ionizing photons absorbed by dust grains (e.g., see the Appendix of McKee \& Williams 1997, who suggest a value of 0.37). To better characterize the ionizing luminosity we also keep track of $\mathrm{Q}\left(\mathrm{He}^{0}\right)$ and $\mathrm{Q}\left(\mathrm{He}^{1}\right)$ which represent the numbers of ionizing photons in the He I and He II continua, respectively.

The above steps allow us to create a discrete two-dimensional table for each flux band where one axis represents stellar mass, the other represents time, and the value of the table is the logarithm of the flux in that band at the appropriate mass and time. Our tables are created through use of the isochrone synthesis method such that our results are stable against the numerical issues that arise from a fixed mass approach (Charlot \& Bruzual 1991).

\subsection{Evaluating the Stellar Properties}

To determine the properties of a given star of any mass at any given time, we first determine if the star is still "alive." This
Table 4

\begin{tabular}{|c|c|}
\hline Filter & Reference \\
\hline NUV & 1 \\
\hline FUV & 1 \\
\hline$u$ & 2 \\
\hline$g$ & 2 \\
\hline$r$ & 2 \\
\hline$i$ & 2 \\
\hline$z$ & 2 \\
\hline$J$ & 3 \\
\hline$H$ & 3 \\
\hline$K$ & 3 \\
\hline$U$ & 4 \\
\hline$B$ & 4 \\
\hline$V$ & 4 \\
\hline$R$ & 4 \\
\hline$I$ & 4 \\
\hline $\mathrm{Q}\left(\mathrm{H}^{0}\right)$ & 5 \\
\hline $\mathrm{Q}\left(\mathrm{He}^{0}\right)$ & 5 \\
\hline $\mathrm{Q}\left(\mathrm{He}^{1}\right)$ & 5 \\
\hline$L_{\text {bol }}$ & 6 \\
\hline \multicolumn{2}{|l|}{ Notes. } \\
\hline \multicolumn{2}{|c|}{${ }^{1}$ GALEX: Morrissey et al. (2005). } \\
\hline \multicolumn{2}{|c|}{${ }^{2}$ SDSS: Fukugita et al. (1996). } \\
\hline \multicolumn{2}{|c|}{${ }^{3}$ Skrutskie et al. (2006). } \\
\hline \multicolumn{2}{|c|}{${ }^{4}$ Johnson-Cousins: Appenzeller et al. (1998). } \\
\hline \multirow{2}{*}{\multicolumn{2}{|c|}{$\begin{array}{l}5 \text { Obtained by integrating SED blueward of } \\
912,504 \text {, and } 208 \AA \text { for } \mathrm{Q}\left(\mathrm{H}^{0}\right), \mathrm{Q}\left(\mathrm{He}^{0}\right) \text {, } \\
\mathrm{Q}\left(\mathrm{He}^{1}\right) \text {, respectively. }\end{array}$}} \\
\hline & \\
\hline${ }^{6}$ Given & cks. \\
\hline
\end{tabular}

Broadband Filters

is done by an interpolation in time to find the minimum mass of a dead star $\left(m_{\text {death }}\right)$ at a given time according to our stellar evolution models (where we call a star "dead" if it no longer has entries in our stellar tracks). If the star is less massive than $m_{\text {death }}$, we interpolate our model tables to determine the flux in a given filter to a precision of 0.01 dex.

For computational speed, there are a variety of approximations and restrictions we are forced to implement. The current scheme only allows ages up to $1 \mathrm{Gyr}$ for the stellar tracks (to be expanded in later releases of the code). We do not evolve stars less massive than $0.9 M_{\odot}$ (a number which can be changed by the user). These stars do not evolve past the main sequence for the current maximum age of the code of $1 \mathrm{Gyr}$, so these stars are treated as having their zero-age main-sequence properties at all times. Due to limitations of the stellar tracks, we treat the photometric properties of all stars less massive than $0.156 M_{\odot}$ identically to those of $0.156 M_{\odot}$ stars. For many purposes, more massive stars dominate the light in the bands such that this approximation is reasonable (but caution is advised for redder bands dominated by older populations). The tracks also impose a $120 M_{\odot}$ upper mass limit on stars.

Currently, we neglect the effects of binary stellar evolution (see Eldridge \& Stanway 2009; van Bever \& Vanbeveren 1998; Dionne \& Robert 2006), which may have an impact on the derived results by producing a bluer population with a reduced number of red supergiants and increased age range of Wolf-Rayet stars.

\subsection{Cluster Disruption}

If the user chooses to form stars in star clusters, we randomly disrupt our clusters in a mass-independent way such that 

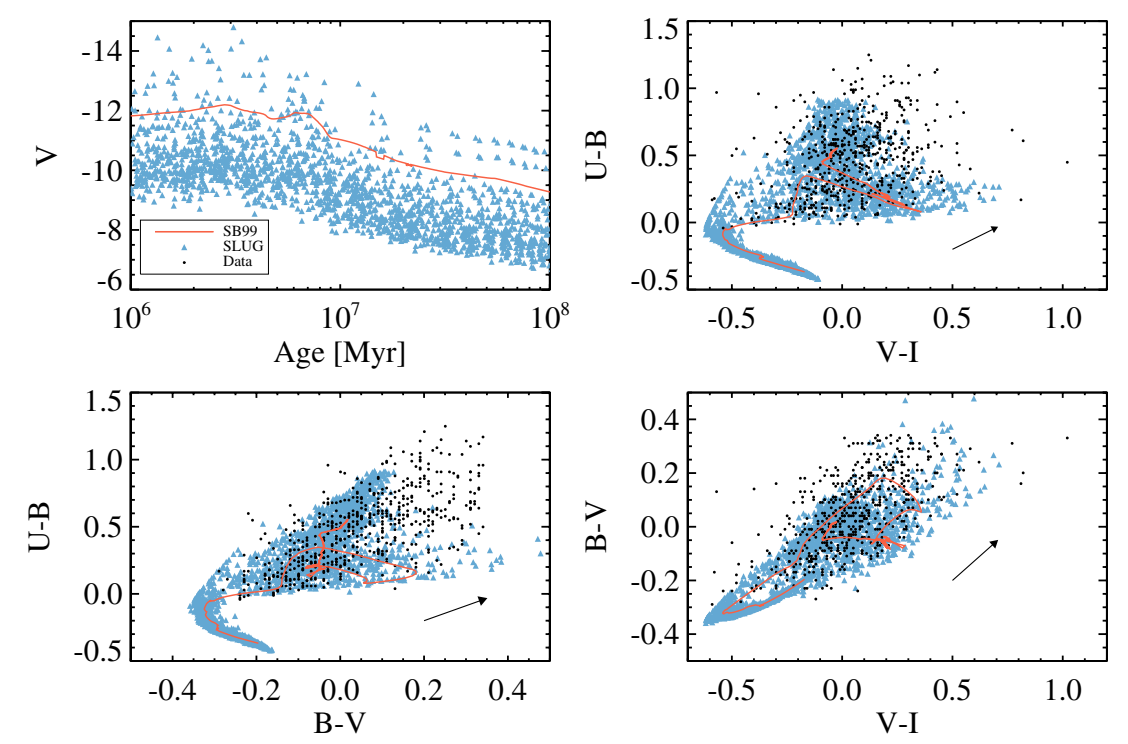

Figure 4. Comparison of observed young star clusters from Larsen (1999; black points) to SLUG models of clusters $>10^{4} M_{\odot}$ (blue triangles). The orange curves show the trajectory of an SB99 $10^{5} M_{\odot}$ cluster as a function of time. Data are omitted from upper left panel as the ages are not present in the Larsen (1999) catalog. Arrows denote the extinction vector for $A_{V}=0.5 \mathrm{mag}$ (created following Appendix B of Schlegel et al. 1998).

(A color version of this figure is available in the online journal.)

Table 5

Fiducial Inputs

\begin{tabular}{lc}
\hline \hline Parameter & Fiducial Value \\
\hline Time step & $10^{6} \mathrm{yr}$ \\
Maximum time & $10^{9} \mathrm{yr}$ \\
IMF & $1-120 M_{\odot} ;$ slope $=-2.35$ \\
ICMF & $20-10^{7} M_{\odot} ;$ slope $=-2$ \\
Stellar evolutionary tracks & Padova+AGB \\
Metallicity & Solar; $Z=0.20$ \\
Stellar atmosphere & Lej+Smi \\
Fraction of stars in clusters & $100 \%$ \\
\hline
\end{tabular}

Notes.

a While the preferred SEDs for SB99 are the Pau+Smi atmospheres, we find that the Pauldrach models are far too discrete. Therefore, while we provide the Pau+Smi atmospheres, we recommend the Lej+Smi.

$d N / d \tau \propto \tau^{-1}$ (following Fall et al. 2009). We start cluster disruption 1 Myr after the cluster forms. This results in $90 \%$ of star clusters being disrupted for each factor of 10 in age after 1 Myr. We continue to calculate the photometry for stars in disrupted clusters, and we include their contribution in our calculations of the integrated properties of the galaxy, as well as in a set of "field" variables and outputs.

\section{VALIDATING TESTS}

In this section we present a variety of tests to validate the outputs of SLUG. For these tests we make use of a set of fiducial parameters presented in Table 5 unless otherwise noted. ${ }^{8}$ To emphasize that SLUG can be applied at different regimes, we arrange these tests in order of scale starting with individual clusters and then considering integrated properties of entire galaxies in the well-sampled regime.

\footnotetext{
8 Since we aim to test SLUG rather than to perform a study of the effects that the multiple parameters have on the luminosity distributions, we choose widely adopted values.
}

\subsection{Photometry of Clusters}

To demonstrate that SLUG reproduces properties of observed clusters, we turn to the catalog of young star clusters compiled in Larsen (1999). To reproduce the clusters' photometry we modify our fiducial IMF to extend down to $0.08 M_{\odot}$ and run a SLUG model with an SFR of $1 M_{\odot} \mathrm{yr}^{-1}$ for $500 \mathrm{Myr}$, evaluated every 10 Myr. Note that the SFR does not directly affect the ICMF or the properties of the clusters, only the number of clusters in existence at a given time. We show the results of this exercise in Figure 4 where we find remarkable agreement between the models and the data. As is clear from the figure, we are able to reproduce both the location and spread of most of the observed data. Clusters that fall outside of the locus of the SLUG models can easily be reproduced when one accounts for a modest amount of reddening (see reddening vector).

\subsection{Cluster Birthline}

Another test of the photometry of clusters is to compare their $\mathrm{H} \alpha$ luminosity to their bolometric luminosity. Work by Corbelli et al. (2009) has shown that newly born clusters lie along a birthline in this parameter space. They found that the distribution of star clusters was incompatible with a truncation of the IMF within clusters determined by the mass of the clusters are prescribed by Weidner \& Kroupa (2006). In Figure 5 we compare the same models as in Section 4.1 (assuming $f_{\text {esc }}=0$ and $f_{\text {dust }}=0$ ) with those of Corbelli et al. (2009), and find good agreement without altering the IMF. Our theoretical predictions differ slightly in the tilt of the locus of points from those by Corbelli et al. (2009), since we characterize the properties of our stars in a different manner (making use of stellar tracks rather than fitting formulae). To better demonstrate the origin of the birthline we also make use of SLUG's ability to keep track of the IMF of each individual cluster (see bottom panel of Figure 5). We group clusters by their location in this diagram and sum all of their IMFs together to produce a mean IMF for each region. Here we can see that the birthline (from left to right) is a sequence of clusters with progressively more well-sampled upper ends of the IMF. Extremely rare deviants exist below the 


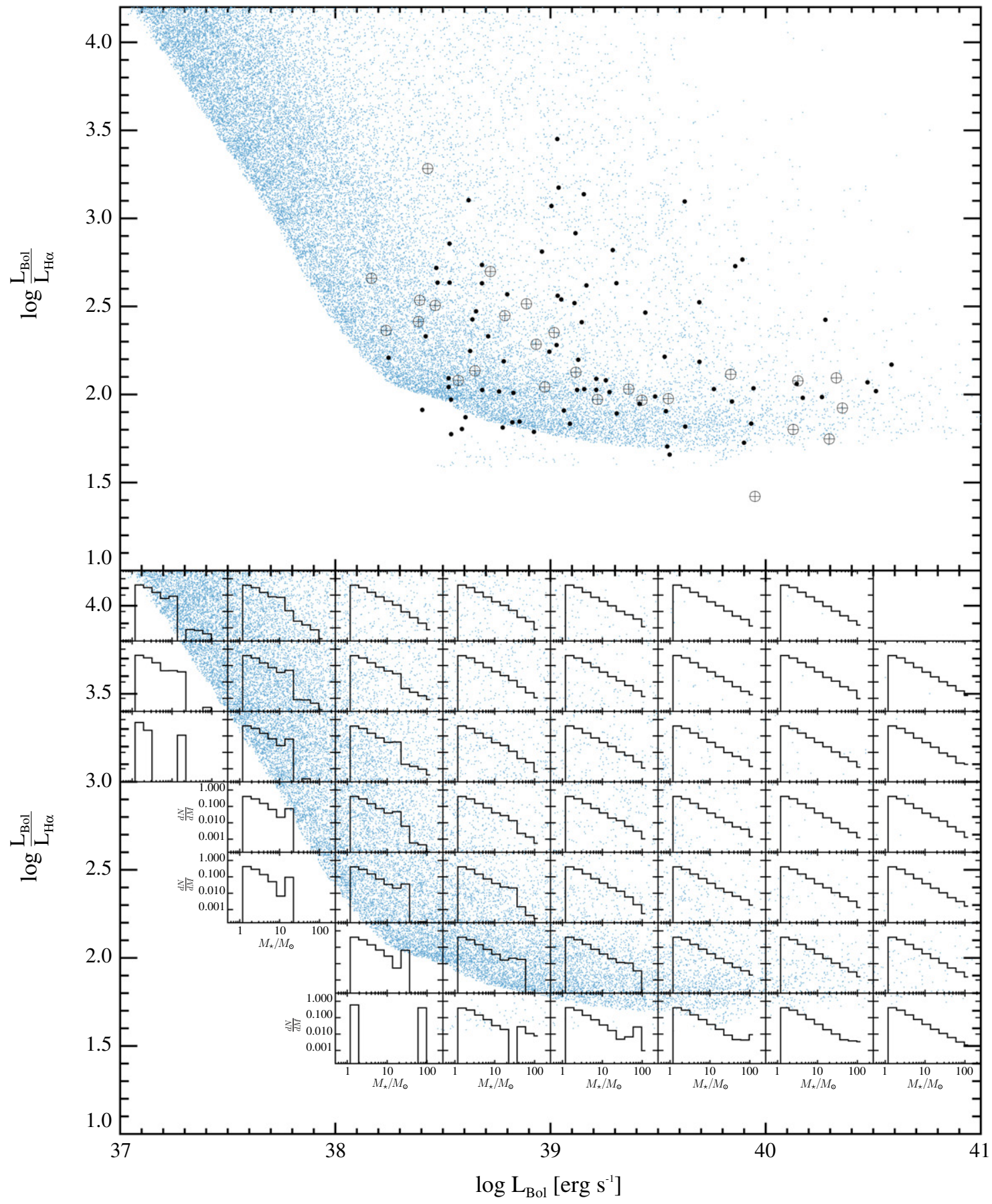

Figure 5. (top) Here we present the birthline as first discussed by Corbelli et al. (2009) whose original data are shown as black points and crosses (circle-crosses denote their "clean" sample). Blue data points are clusters from SLUG. We see that our models are in relatively good agreement with observations. (bottom) We present overlays demonstrating the average IMFs in each region of the birthline plot. Note how the IMF becomes progressively more bottom heavy moving to lower luminosity.

(A color version of this figure is available in the online journal.)

birthline where more extremely massive $\left(>100 M_{\odot}\right)$ stars are drawn than average. Note that these rare clusters consisting of essentially isolated $\mathrm{O}$ stars have been reported in the Milky Way (de Wit et al. 2004, 2005) and the SMC (Oey et al. 2004; Lamb et al. 2010) in numbers consistent with stochastic sampling of the IMF. This prediction of the preferential combinations of IMF realizations lying along the cluster birthline will be testable with future observations and is an example of the predictive power and insight provided by SLUG.

\subsection{Comparison with SB99}

A third obvious comparison for SLUG is SB99 itself. Being widely used, SB99 serves as a benchmark for our code. Indeed, one of the motivations for making use of the same tracks and SED algorithms as SB99 is that our code should be able to exactly reproduce SB99 in the well-sampled regime. To that end we now present a variety of tests where we compare to SB99 to demonstrate to demonstrate that we can reproduce their results 


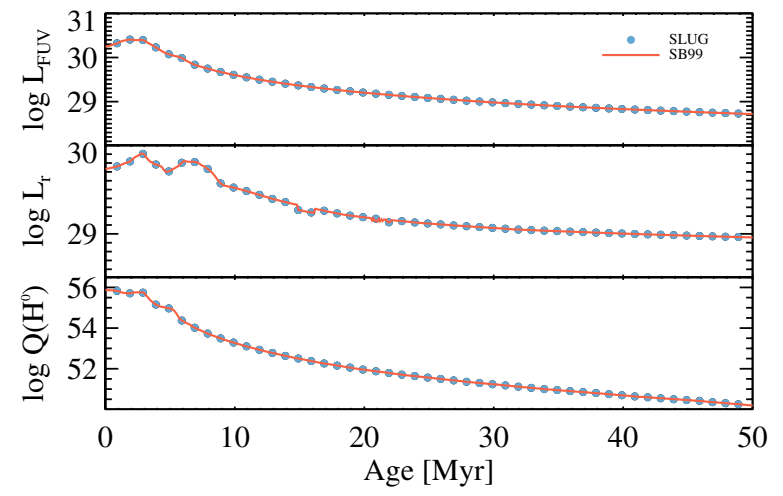

Figure 6. Comparison of SLUG and SB99 simulations of an instantaneous burst of $10^{9} M_{\odot}$. We find good agreement between the two predictions in both the absolute normalization of the fluxes as well as the time-dependent behavior. FUV and $r$-band fluxes are in units of $\operatorname{erg~s}^{-1} \mathrm{~Hz}^{-1}$ while $\mathrm{Q}\left(\mathrm{H}^{0}\right)$ is in units of photons $\mathrm{s}^{-1}$. In the $L_{r}$ panel, in the age ranges of 12-18 Myr and 20-22 Myr, one can see minor imperfections in interpolating the stellar tracks as implemented by SB99.

(A color version of this figure is available in the online journal.)

in the regime where neither sampling nor temporal stochasticity are important.

To compare the outputs of both SB99 and SLUG, we choose an instantaneous burst of star formation to demonstrate the matching of the codes in both amplitude and time. We run an SB99 model similar to our fiducial model (i.e., IMF slope of -2.35 from $1-120 M_{\odot}$, solar metallicity, Padova+AGB tracks, and Lej+Smi SEDs; see Table 3 for definitions of these parameters). To meaningfully compare with SB99 we must choose SLUG input parameters such that we are evaluating a population where SB99's estimates of the mean are a valid model for the total luminosity of the system. We therefore draw a very large instantaneous population of $10^{9} M_{\odot}$. To nullify any possible effects of random truncations due to populating the clusters, we ensure all clusters are very large by modifying the fiducial ICMF to a restricted range $\left(10^{6}\right.$ to $\left.2 \times 10^{6} \mathrm{M}_{\odot}\right)$. Similar results are obtained if we simply turn clustering off. We present the results in Figure 6. It is evident that we are accurately able to reproduce SB99 in the well-sampled regime for integrated "galaxy" properties. We match both the amplitude and time evolution in all photometric bands.

This can also be seen by looking at the full SEDs. In Figure 7, we present photometry for all 15 of the flux bands available for SLUG and compare with the spectra and integrated photometry produced by SB99 at a variety of time steps. Again we are able to fully reproduce the photometric properties in the well-sampled regime from FUV to $K$ band.

In both these tests, SLUG matches SB99 within 0.026 dex for all fluxes at all times.

\section{STOCHASTICITY IN ACTION}

Having demonstrated that SLUG can reproduce realistic clusters as well as reproduce SB99's results, we now present outputs of SLUG in the stochastic regime.

\subsection{Effects on Coeval Populations}

Recent studies (e.g., Dalcanton et al. 2009) have demonstrated the wealth of information that can be obtained using resolved CMDs of stars within a galaxy. For comparison with such studies in the stochastic regime, SLUG produces two-dimensional histograms for the user's choice of filters. Such diagrams allow us to directly characterize the effects of stochasticity in a coeval population. In Figure 8, we compare two realizations of CMDs produced by SLUG for a $10^{5} M_{\odot}$ instantaneous burst to the theoretical isochrones from which they are produced. Aside from demonstrating that we accurately reproduce the tracks, we are able to see the effects of stochasticity in populating the rapid phases of evolution differently in the two realizations. Note that SLUG is capable of producing such diagrams for any given SFH.

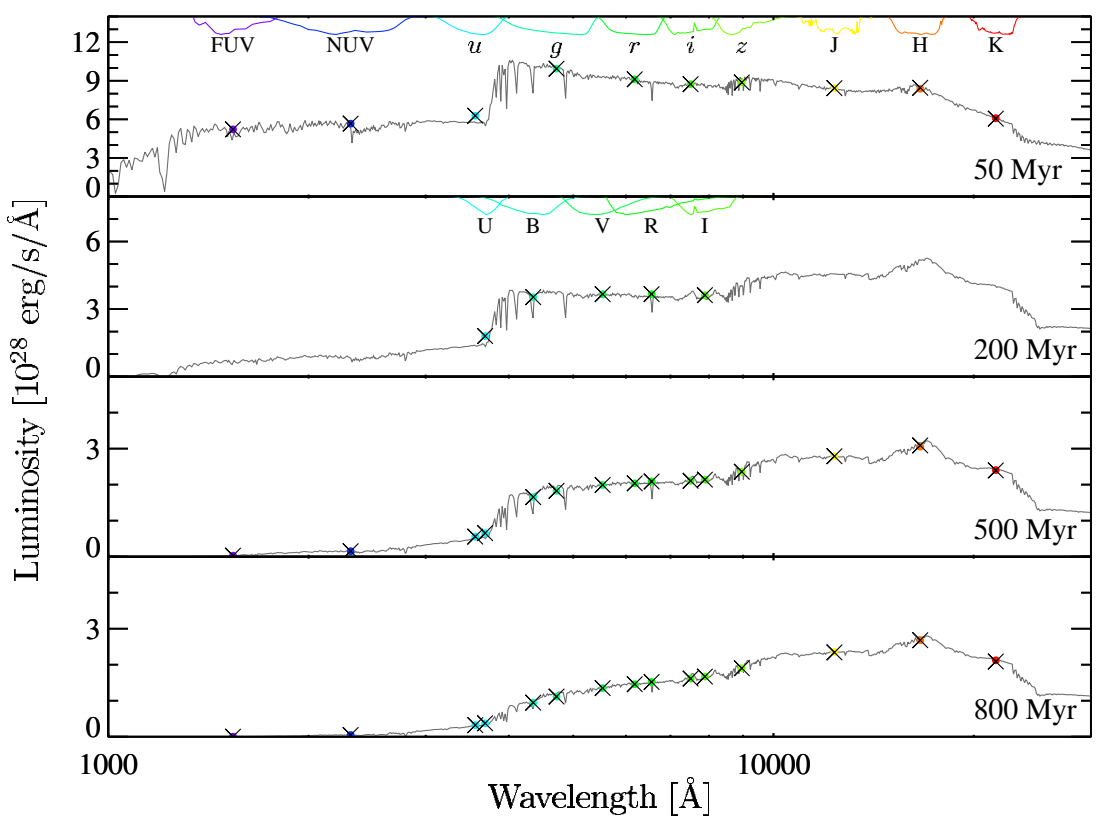

Figure 7. Comparison of SLUG and SB99 photometry for an instantaneous burst of $10^{9} M_{\odot}$, evaluated at the ages indicated in each panel. The gray solid line represents the output spectrum from SB99 for such a population. The filled color circles show the SB99 integrated fluxes for the filters available to SLUG. The black $X$ 's mark the SLUG photometry for the well-sampled model described in Section 4.3.

(A color version of this figure is available in the online journal.) 

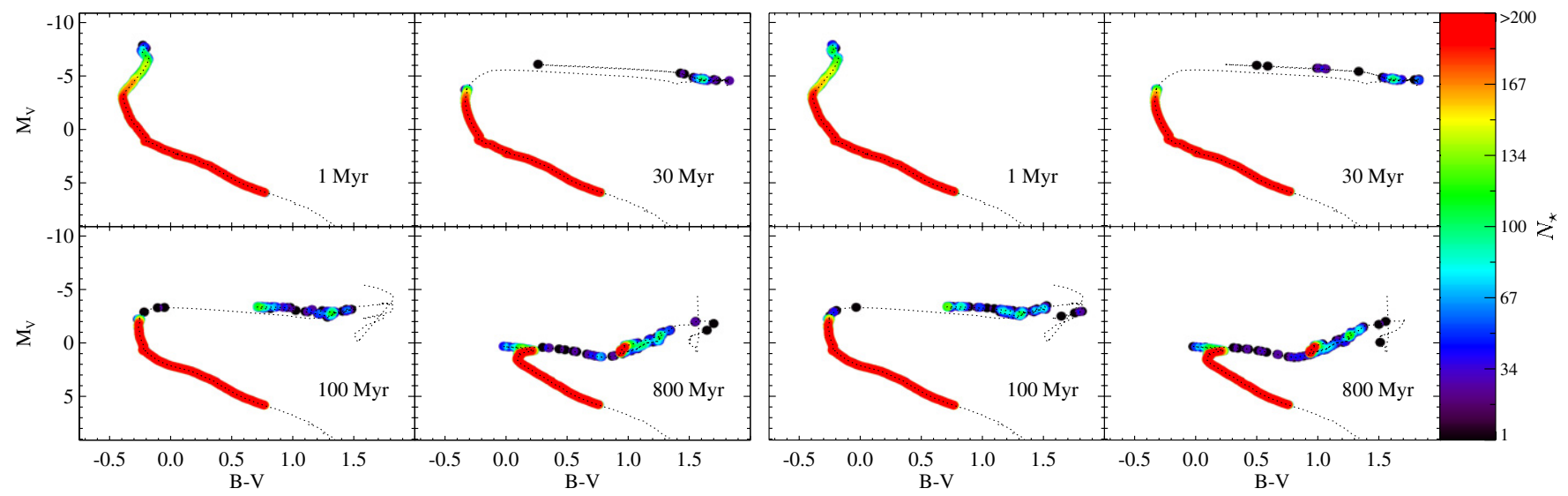

Figure 8. CMDs of two realizations of an instantaneous $10^{5} M_{\odot}$ burst population at the ages indicated in each panel. Only stars more massive than $0.9 M_{\odot}$ are binned in the CMDs. The dotted lines show the corresponding theoretical isochrones. The SLUG CMD has been convolved with circular top-hat point-spread function solely to improve visibility. The color bar denotes the number of stars in that region of the diagram.

(A color version of this figure is available in the online journal.)
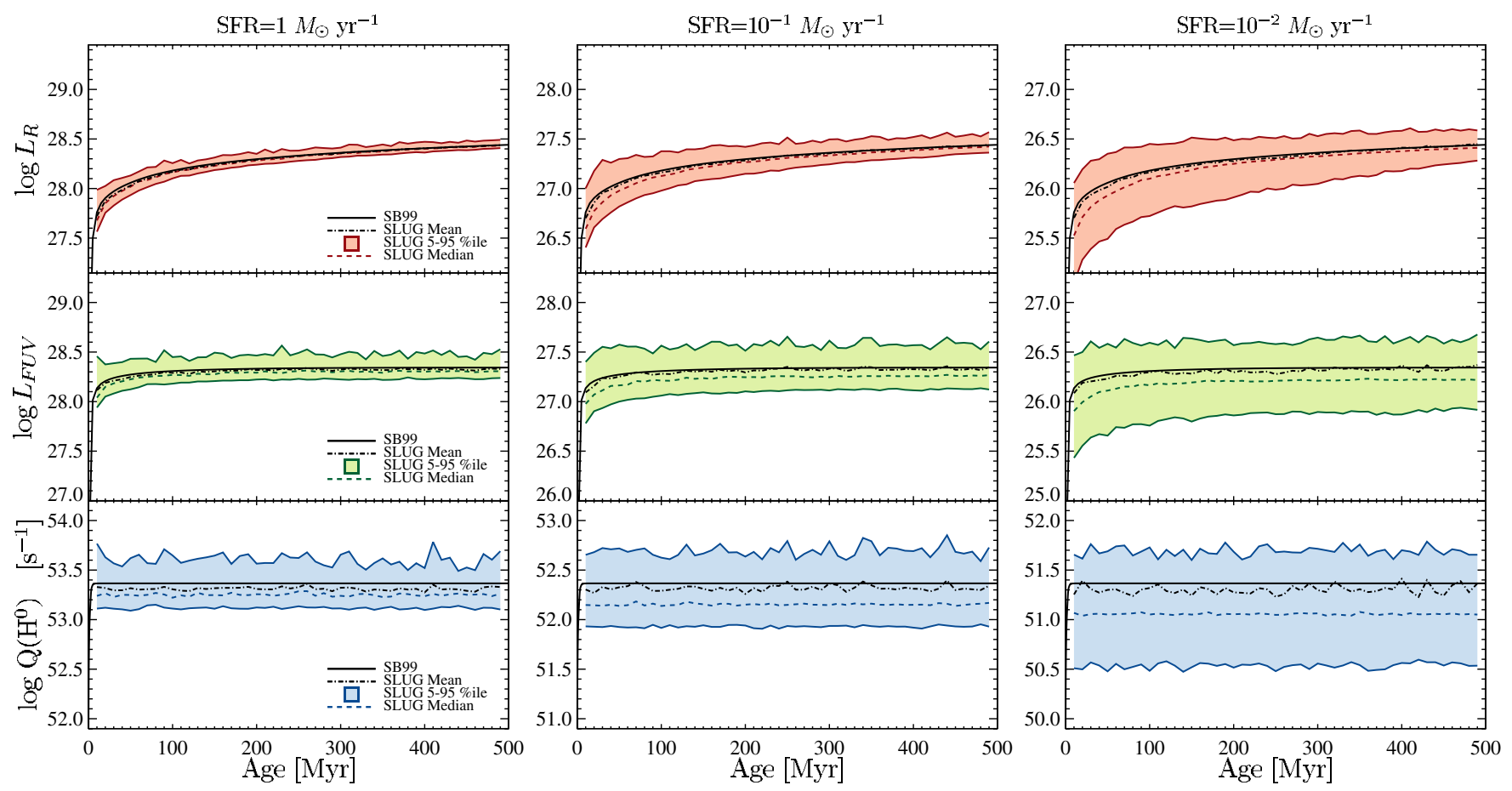

Figure 9. $R$-band, FUV, and ionizing photon luminosities vs. time for galaxies with constant SFRs of $1,10^{-1}$, and $10^{-2} M_{\odot} \mathrm{yr}^{-1}$ as indicated. $R$-band and FUV luminosities are in units of $\mathrm{erg} \mathrm{s}^{-1} \mathrm{~Hz}^{-1}$. We compare a fully sampled realization from SB99 (solid black lines) with 100, 500, and 1000 realizations from SLUG for SFRs of $1,10^{-1}$, and $10^{-2} M_{\odot} \mathrm{yr}^{-1}$, respectively. The SLUG models are represented by their arithmetic mean (black dash-dotted line), median (colored dashed line), and 5-95 percentile range (filled color region). The time step in our SLUG models was set to 10 Myr. Note that the $y$-axis in each panel has been chosen to match the SFR, but always spans the same logarithmic interval.

(A color version of this figure is available in the online journal.)

\subsection{Effects on Composite Populations}

While individual clusters of stars can be treated as coeval, larger systems are intrinsically built of composite populations. One of the most basic composite populations one can consider is a galaxy forming stars at a constant SFR. As discussed in Section 2.2, the value of the SFR will have a significant impact on the effects of stochasticity.

To demonstrate the differences that stochasticity makes, we compare SLUG realizations to those of a well-sampled SB99 model. In Figure 9, we first examine the luminosities for SFRs of 100,500 , and 1000 realizations of $1,10^{-1}$, and $10^{-2} M_{\odot} \mathrm{yr}^{-1}$, respectively. We use our fiducial values for the ICMF and cluster mass fraction. For each SFR, we show the mean and median of the SLUG runs along with the 5 and 95 percentiles.

First, we note that the agreement between the mean of the SLUG models and the SB99 prediction of the mean again exhibits our ability to reproduce SB99's outputs.

However, one can clearly see an increase in fractional scatter as the SFR decreases. This can be attributed to the more bursty SFHs which are the result of the grouping of age in massive clusters. This scatter appears at higher SFRs than predicted by our naive discussion in Section 2.2 as a direct result of the clustering. In fact, nearly all of the scatter seen in Figure 9 is 

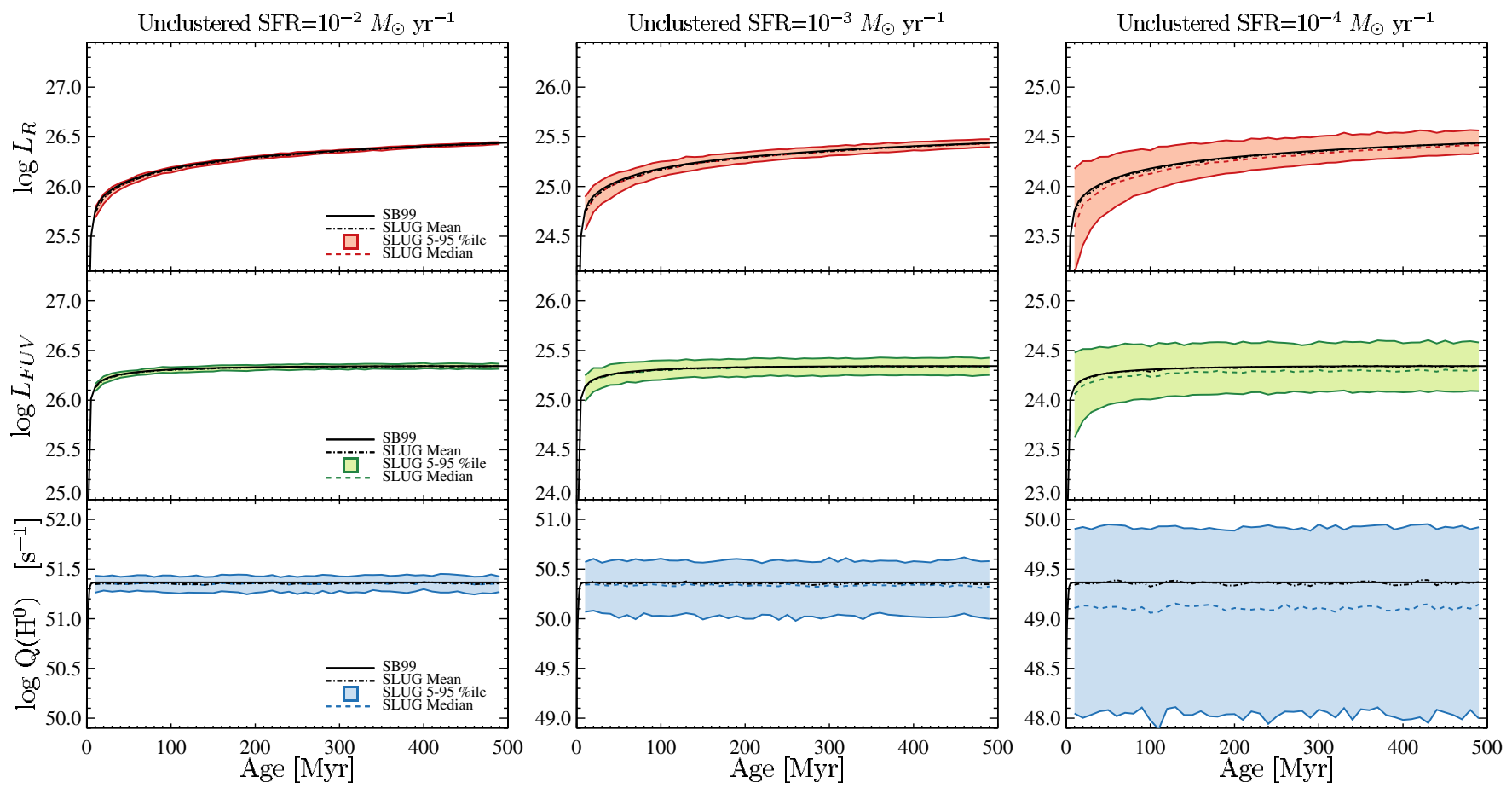

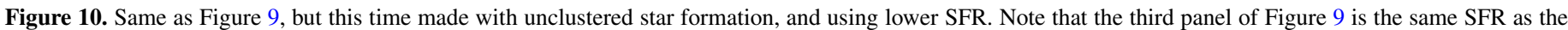
first panel of this figure. These figures were constructed with 100, 500, and 1000 realizations at SFRs of $10^{-2}, 10^{-3}$, and $10^{-4} M_{\odot} \mathrm{yr}^{-1}$, respectively.

(A color version of this figure is available in the online journal.)

a result of the clustering rather than sampling of the IMF in clusters. This is most clearly demonstrated by Figure 10, which shows similar simulations but with completely unclustered star formation. The figure presents the results of 100,500 , and 1000 realizations of SFRs of $10^{-2}, 10^{-3}$, and $10^{-4} M_{\odot} \mathrm{yr}^{-1}$, respectively. Without clustering the $10^{-2} M_{\odot} \mathrm{yr}^{-1}$ models have approximately an order of magnitude less scatter in the log of the luminosity within the 5-95 percentile range. We see that the unclustered stochastic effects behave as predicted in Section 2.2 where the fractional scatter is small for SFRs $\sim 10^{-2} M_{\odot} \mathrm{yr}^{-1}$ and quickly increases as the SFR decreases (also discussed in Fumagalli et al. 2011a).

For a demonstration of the effects of clustering on the photometric properties of galaxies, we present the tracks of a random subset of individual stochastic realizations of clustered star formation in Figure 11. One can see that the $\mathrm{Q}\left(\mathrm{H}^{0}\right)$ curves are less uniform than the $R$ luminosity. This is a direct result of the sensitivity of $\mathrm{Q}\left(\mathrm{H}^{0}\right)$ to the youngest, most massive stars. One can also see that the scatter increases with decreasing SFR as expected. Of note is the evolution of the 4th from the top simulation with SFR $=10^{-2} M_{\odot} \mathrm{yr}^{-1}$ as it is marked by several significantly large clusters which lead to a very "bursty" SFH. This is to be further discussed in R. L. da Silva et al. (2011, in preparation) where we elaborate on the effects of stochastic star formation when one includes clusters.

\section{SUMMARY}

We introduce SLUG, a new code that correctly accounts for the effects of stochasticity (with caveats discussed in the text) by populating galaxies with stars and clusters of stars and then following their evolution using stellar evolutionary tracks. Cluster disruption is taken into account and a variety of outputs are created.
We present a series of tests comparing SLUG to observations and other theoretical predictions. SLUG is able to reproduce the photometric properties of clusters from the Larsen (1999) catalog as well as the Corbelli et al. (2009) birthline. It can also reproduce the results of SB99 in the well-sampled regime.

Finally, we present SLUG outputs in the stochastic regime and demonstrate the flexibility of the code to address a variety of astrophysical problems with its variety of possible outputs.

SLUG is a publicly available code and can be found at http://sites.google.com/site/runslug/.

We thank the anonymous referee for useful comments which improved the clarity of the paper. The work of R.L.dS. is supported by a National Science Foundation Graduate Research Fellowship. R.L.dS. is partially supported by an NSF CAREER grant (AST-0548180). M.R.K. acknowledges support from: an Alfred P. Sloan Fellowship; the National Science Foundation through grants AST-0807739 and CAREER-0955300; and NASA through Astrophysics Theory and Fundamental Physics grant NNX09AK31G and a Spitzer Space Telescope theoretical research program grant. We thank J. X. Prochaska for help in reading and providing input on the early stages of this manuscript. We thank F. Bigiel for encouraging us to create SLUG and useful conversations with J. Eldridge, C. Weidner, R. Bernstein, and J. Colucci.

\section{APPENDIX IMPLEMENTATION OF IGIMF}

The IGIMF theory (Kroupa \& Weidner 2003; Weidner et al. 2011) is a statement that (1) most (if not all) stars form in clusters, (2) the SFR controls the upper cutoff of the ICMF, and (3) that each cluster's mass changes the upper cutoff of the 

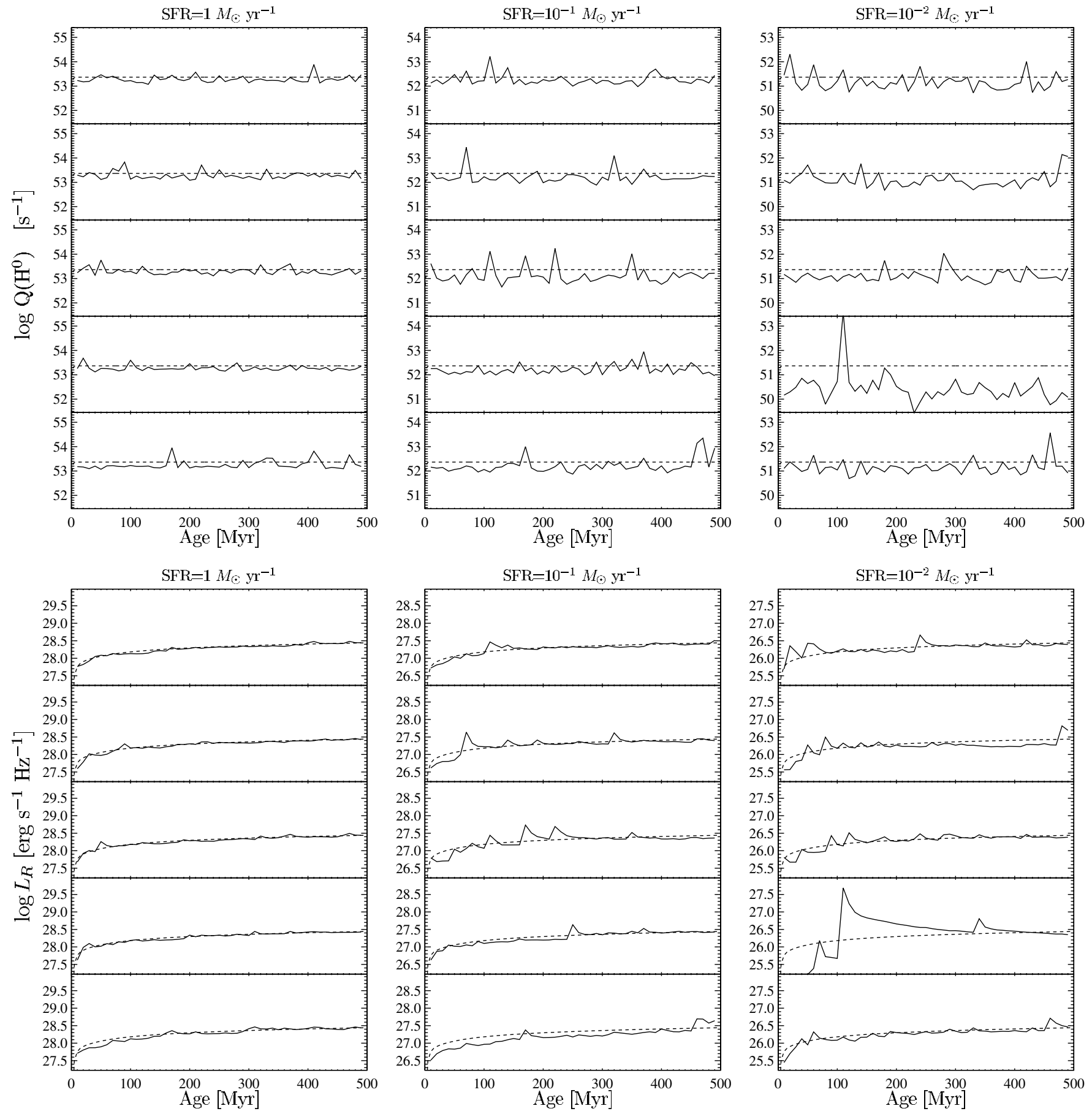

Figure 11. Solid lines show the evolution of $\mathrm{Q}\left(\mathrm{H}^{0}\right)$ and $R$-band luminosity for individual simulations with clustered star formation with SFRs of 1 , $10^{-1}$, and $10^{-2} M_{\odot} \mathrm{yr}^{-1}$. Dashed lines show the SB99 prediction. Note that the $y$-axis in each panel has been chosen to match the SFR, but always spans the same logarithmic interval.

IMF in that cluster. Thus, the distribution of stars in a galaxy is not simply drawn from the IMF, but is the result of a joint distribution function of stars and star clusters.

We implement the IGIMF following Weidner et al. (2011). We use the work of Pflamm-Altenburg \& Kroupa (2008), Weidner \& Kroupa (2005), and Weidner et al. (2004) to define the maximum cluster mass as

$$
M_{\mathrm{ecl}, \max }=84793\left(\frac{\langle\mathrm{SFR}\rangle}{M_{\odot} \mathrm{yr}^{-1}}\right)^{3 / 4}
$$

where $\langle\mathrm{SFR}\rangle$ is the time-average SFR. Thus the SFR affects the upper cutoff of the ICMF. We determine the average SFR over a time interval defined by the user (fiducially $\left.10^{7} \mathrm{yr}\right)$.

After a cluster mass has been drawn, we must adjust the upper cutoff of the IMF that we use to draw stars for that cluster. The relation between maximum stellar mass and cluster mass $\left(m_{\max }-M_{\text {ecl }}\right)$ has been studied by Weidner \& Kroupa (2004) and Weidner et al. (2010). Following their treatment, we solve a system of equations numerically for $m_{\max }$ as a function of $M_{\mathrm{ecl}}$. 

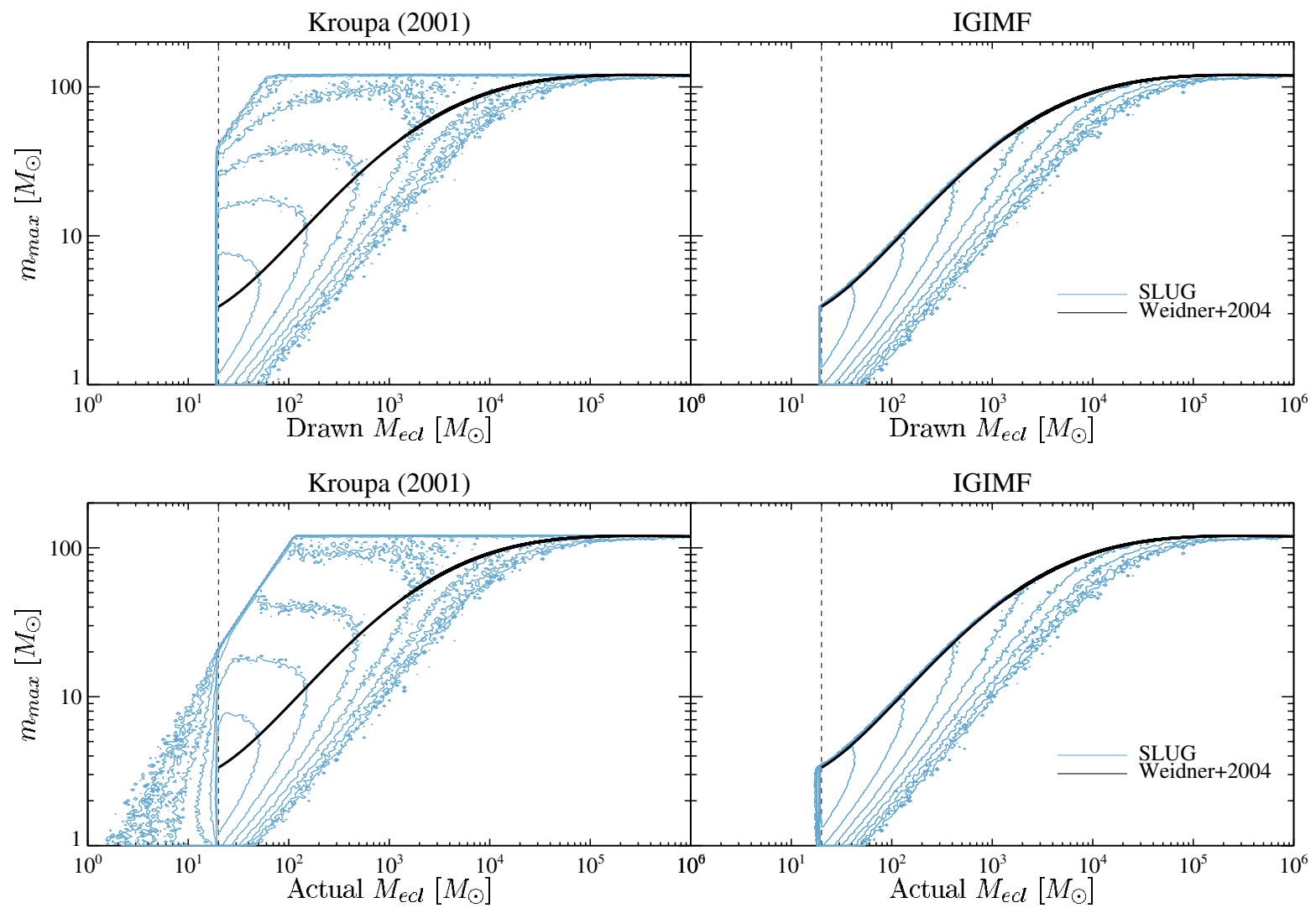

Figure 12. Mass of the largest star in a cluster vs. that cluster's mass for clusters created by SLUG for a Kroupa (2001) IMF (left) and the IGIMF (right). The black lines denote the analytic prediction of the maximum possible stellar mass in a cluster in the IGIMF model, the black dashed line notes the lower limit of the initial cluster mass function, and blue contours denote the location of SLUG models. Top panels show the maximum stellar mass as a function of the cluster mass drawn from the ICMF, while bottom panels show the same relation relative to the sum of the masses of all stars actually populating the clusters. These two differ slightly-see Section 3.2 .

(A color version of this figure is available in the online journal.)

The first equation is simply a statement that the total cluster mass $\left(M_{\text {ecl }}\right)$ is the integral of the distribution of masses $\left(\frac{d N}{d m}\right)$ integrated from the lowest to highest mass star in the cluster:

$$
M_{\mathrm{ecl}}=\int_{m_{\min }}^{m_{\max }} m \frac{d N}{d m} d m
$$

The next constraint is derived based on the statement that there is only one star in the cluster with mass equal to $m_{\max }$. Their choice of implementation of this statement is as follows: ${ }^{9}$

$$
1=\int_{m_{\max }}^{m_{\max , \star}} \frac{d N}{d m} d m,
$$

where $m_{\max , \star}$ is the maximum stellar mass possible.

In the specific case of a Kroupa (2001) IMF, these equations reduce to the following (taken from Weidner \& Kroupa 2004).

$$
1=k\left[\left(\frac{m_{\mathrm{H}}}{m_{0}}\right)^{\alpha_{1}}\left(\frac{m_{0}}{m_{1}}\right)^{\alpha_{2}} m_{1}^{\alpha_{3}}\left(\frac{m_{\max , *}^{1-\alpha^{3}}}{1-\alpha_{3}}-\frac{m_{\max }^{1-\alpha^{3}}}{1-\alpha_{3}}\right)\right]
$$

\footnotetext{
9 Cerviño et al. (2011) have pointed out that this expression does not equate to the logical statement mentioned above - it underpredicts the maximum mass in $63 \%$ of cases. In fact, this formalism equates rather to the statement that the expectation value of stars in the interval $m_{\max }-m_{\max , \star}$ is equal to 1 . However, this is the standard formalism of the IGIMF, so it is the formalism we implement.
}

$$
\begin{aligned}
\frac{M_{\mathrm{cl}}}{k}= & \frac{m_{\mathrm{H}}^{\alpha_{0}}}{2-\alpha_{0}}\left(m_{\mathrm{H}}^{2-\alpha_{0}}-m_{\mathrm{low}}^{2-\alpha_{0}}\right)+\frac{m_{\mathrm{H}}^{\alpha_{1}}}{2-\alpha_{1}}\left(m_{0}^{2-\alpha_{1}}-m_{\mathrm{H}}^{2-\alpha_{1}}\right) \\
& +\frac{\left(\frac{m_{\mathrm{H}}}{m_{0}}\right)^{\alpha_{2}} m_{0}^{\alpha_{2}}}{2-\alpha_{1}}\left(m_{1}^{2-\alpha_{2}}-m_{0}^{2-\alpha_{2}}\right) \\
& +\frac{\left(\frac{m_{\mathrm{H}}}{m_{0}}\right)^{\alpha_{2}}\left(\frac{m_{0}}{m 1}\right)^{\alpha_{2}} m_{1}^{\alpha_{3}}}{2-\alpha_{3}}\left(m_{\max }^{2-\alpha_{3}}-m_{0}^{2-\alpha_{3}}\right),
\end{aligned}
$$

where

$$
\begin{array}{ll}
\alpha_{0}=+0.30, & m_{\text {low }}=0.01 \\
\alpha_{1}=+1.30, & m_{\mathrm{H}}=0.08 \\
\alpha_{2}=+2.30, & m_{0}=1.00 \\
\alpha_{3}=+2.35, & m_{\text {max }, *}=120 .
\end{array}
$$

We fit a sixth-order polynomial to the numerical solution to find

$$
\log _{10} m_{\max }=\sum_{i=0}^{6} a_{i}\left(\log _{10} M_{\mathrm{cl}}\right)^{i},
$$

where $a=[1.449,-2.522,2.055,-0.616,0.0897,-0.00643$, $0.000182]$.

We then use this upper mass limit to modify the standard Kroupa (2001) IMF to fill in the stars for each cluster. Figure 12 demonstrates the result. One can see that we are accurately applying the cutoff to the IMF in the IGIMF. 


\section{REFERENCES}

Appenzeller, I., Fricke, K., Fürtig, W., et al. 1998, Messenger, 94, 1 Bernasconi, P. A., \& Maeder, A. 1996, A\&A, 307, 829

Bigiel, F., Leroy, A., Walter, F., et al. 2010, AJ, 140, 1194

Boissier, S., Gil de Paz, A., Boselli, A., et al. 2007, ApJS, 173, 524

Boissier, S., Gil de Paz, A., Boselli, A., et al. 2008, ApJ, 681, 244

Boselli, A., Boissier, S., Cortese, L., et al. 2009, ApJ, 706, 1527

Brocato, E., Castellani, V., Raimondo, G., \& Romaniello, M. 1999, A\&AS, 136, 65

Buzzoni, A. 1989, ApJS, 71, 817

Cerviño, M., \& Luridiana, V. 2004, A\&A, 413, 145

Cerviño, M., \& Luridiana, V. 2006, A\&A, 451, 475

Cerviño, M., Luridiana, V., Pérez, E., Vílchez, J. M., \& Valls-Gabaud, D. 2003, A\&A, 407, 177

Cerviño, M., Perez, E., Sanchez, N., Roman-Zuniga, C., \& Valls-Gabaud, D. 2011, in ASP Conf. Proc. 440, UP2010: Have Observations Revealed a Variable Upper End of the Initial Mass Function?, ed. M. Treyer et al. (San Francisco, CA: ASP), 133

Cerviño, M., \& Valls-Gabaud, D. 2003, MNRAS, 338, 481

Chabrier, G. 2003, PASP, 115, 763

Chandar, R., Fall, S. M., \& Whitmore, B. C. 2010, ApJ, 711, 1263

Charbonnel, C., Däppen, W., Schaerer, D., et al. 1999, A\&AS, 135, 405

Charbonnel, C., Meynet, G., Maeder, A., \& Schaerer, D. 1996, A\&AS, 115, 339

Charlot, S., \& Bruzual, A. G. 1991, ApJ, 367, 126

Colucci, J. E., Bernstein, R. A., Cameron, S. A., \& McWilliam, A. 2011, ApJ, 735,55

Conroy, C., \& Gunn, J. E. 2010, ApJ, 712, 833

Conroy, C., Gunn, J. E., \& White, M. 2009, ApJ, 699, 486

Conroy, C., White, M., \& Gunn, J. E. 2010, ApJ, 708, 58

Corbelli, E., Verley, S., Elmegreen, B. G., \& Giovanardi, C. 2009, A\&A, 495, 479

Cordier, D., Pietrinferni, A., Cassisi, S., \& Salaris, M. 2007, AJ, 133, 468

Crowther, P. A., Schnurr, O., Hirschi, R., et al. 2010, MNRAS, 408, 731

Dalcanton, J. J., Williams, B. F., Seth, A. C., et al. 2009, ApJS, 183, 67

de Jager, C., Nieuwenhuijzen, H., \& van der Hucht, K. A. 1988, A\&AS, 72, 259

de Wit, W. J., Testi, L., Palla, F., Vanzi, L., \& Zinnecker, H. 2004, A\&A, 425, 937

de Wit, W. J., Testi, L., Palla, F., \& Zinnecker, H. 2005, A\&A, 437, 247

Dionne, D., \& Robert, C. 2006, ApJ, 641, 252

Eldridge, J. J. 2011, arXiv:1106.4311

Eldridge, J. J., \& Stanway, E. R. 2009, MNRAS, 400, 1019

Elmegreen, B. G. 2000, ApJ, 539, 342

Elson, R. A. W., Fall, S. M., \& Freeman, K. C. 1989, ApJ, 336, 734

Fagotto, F., Bressan, A., Bertelli, G., \& Chiosi, C. 1994a, A\&AS, 105, 29

Fagotto, F., Bressan, A., Bertelli, G., \& Chiosi, C. 1994b, A\&AS, 104, 365

Fall, S. M., Chandar, R., \& Whitmore, B. C. 2009, ApJ, 704, 453

Fall, S. M., Krumholz, M. R., \& Matzner, C. D. 2010, ApJ, 710, L142

Figer, D. F. 2005, Nature, 434, 192

Fioc, M., \& Rocca-Volmerange, B. 1997, A\&A, 326, 950

Fouesneau, M., \& Lançon, A. 2010, A\&A, 521, A22

Fukugita, M., Ichikawa, T., Gunn, J. E., et al. 1996, AJ, 111, 1748

Fumagalli, M., da Silva, R. L., \& Krumholz, M. R. 2011a, ApJ, 741, L26

Fumagalli, M., da Silva, R. L., Krumholz, M. R., \& Bigiel, F. 2011b, in ASP

Conf. Ser. 440, UP2010: Have Observations Revealed a Variable Upper End of the Initial Mass Function?, ed. M. Treyer, T. K. Wyder, J. D. Neill, M. Seibert, \& J. C. Lee (San Francisco, CA: ASP), 155

Fumagalli, M., \& Gavazzi, G. 2008, A\&A, 490, 571

Gogarten, S. M., Dalcanton, J. J., Williams, B. F., et al. 2009, ApJ, 691, 115

Haas, M. R., \& Anders, P. 2010, A\&A, 512, A79

Hillier, D. J., \& Miller, D. L. 1998, ApJ, 496, 407

Hirashita, H., Buat, V., \& Inoue, A. K. 2003, A\&A, 410, 83

Hosokawa, T., Offner, S. S. R., \& Krumholz, M. R. 2011, ApJ, 738, 140
Hoversten, E. A., \& Glazebrook, K. 2008, ApJ, 675, 163

Jeffries, R. D. 2007, MNRAS, 381, 1169

Kotulla, R., Fritze, U., Weilbacher, P., \& Anders, P. 2009, MNRAS, 396, 462

Kroupa, P. 2001, MNRAS, 322, 231

Kroupa, P., \& Weidner, C. 2003, ApJ, 598, 1076

Lada, C. J., \& Lada, E. A. 2003, ARA\&A, 41, 57

Lamb, J. B., Oey, M. S., Werk, J. K., \& Ingleby, L. D. 2010, ApJ, 725, 1886

Lançon, A. 2010, arXiv:1011.4821

Lançon, A., \& Mouhcine, M. 2000, in ASP Conf. Ser. 211, Massive Stellar Clusters, ed. A. Lançon \& C. Boily (San Francisco, CA: ASP), 34

Larsen, S. S. 1999, A\&AS, 139, 393

Lee, J. C., Gil de Paz, A., Tremonti, C., et al. 2009, ApJ, 706, 599

Lee, J. C., Kennicutt, R. C., Funes, J. G. S. J., Sakai, S., \& Akiyama, S. 2007, ApJ, $671, \mathrm{~L} 113$

Leitherer, C., \& Heckman, T. M. 1995, ApJS, 96, 9

Leitherer, C., Robert, C., \& Drissen, L. 1992, ApJ, 401, 596

Leitherer, C., Schaerer, D., Goldader, J. D., et al. 1999, ApJS, 123, 3

Lejeune, T., Cuisinier, F., \& Buser, R. 1997, A\&AS, 125, 229

Lejeune, T., Cuisinier, F., \& Buser, R. 1998, A\&AS, 130, 65

Maeder, A. 1990, A\&AS, 84, 139

Maeder, A., \& Meynet, G. 1987, A\&A, 182, 243

McKee, C. F., \& Williams, J. P. 1997, ApJ, 476, 144

Meurer, G. R., Wong, O. I., Kim, J. H., et al. 2009, ApJ, 695, 765

Meynet, G., Maeder, A., Schaller, G., Schaerer, D., \& Charbonnel, C. 1994, A\&AS, 103, 97

Mollá, M., García-Vargas, M. L., \& Bressan, A. 2009, MNRAS, 398, 451

Morrissey, P., Schiminovich, D., Barlow, T. A., et al. 2005, ApJ, 619, L7

Oey, M. S., King, N. L., \& Parker, J. W. 2004, AJ, 127, 1632

Osterbrock, D. E., \& Ferland, G. J. 2006, Astrophysics of Gaseous Nebulae and Active Galactic Nuclei (2nd ed.; Sansalito, CA: University Science Books)

Palla, F., \& Stahler, S. W. 1999, ApJ, 525, 772

Pauldrach, A. W. A., Hoffmann, T. L., \& Lennon, M. 2001, A\&A, 375, 161

Pflamm-Altenburg, J., \& Kroupa, P. 2008, Nature, 455, 641

Pietrinferni, A., Cassisi, S., Salaris, M., Cordier, D., \& Castelli, F. 2007, in IAU Symp. 241, Stellar Populations as Building Blocks of Galaxies, ed. A. Vazdekis \& R. F. Peletier (Cambridge: Cambridge Univ. Press), 39

Popescu, B., \& Hanson, M. M. 2009, AJ, 138, 1724

Raimondo, G., Brocato, E., Cantiello, M., \& Capaccioli, M. 2005, AJ, 130, 2625

Reines, A. E., Nidever, D. L., Whelan, D. G., \& Johnson, K. E. 2010, ApJ, 708, 26

Salpeter, E. E. 1955, ApJ, 121, 161

Scalo, J. M. 1986, Fundam. Cosm. Phys., 11, 1

Schlegel, D. J., Finkbeiner, D. P., \& Davis, M. 1998, ApJ, 500, 525

Schmutz, W. 1998, in ASP Conf. Ser. 131, Properties of Hot Luminous Stars, ed. I. Howarth (San Francisco, CA: ASP), 119

Silva-Villa, E., \& Larsen, S. S. 2011, A\&A, 529, A25

Skrutskie, M. F., Cutri, R. M., Stiening, R., et al. 2006, AJ, 131, 1163

Smith, L. J., Norris, R. P. F., \& Crowther, P. A. 2002, MNRAS, 337, 1309

Thilker, D. A., Bianchi, L., Meurer, G., et al. 2007, ApJS, 173, 538

Tinsley, B. M. 1980, Fundam. Cosm. Phys., 5, 287

Vázquez, G. A., \& Leitherer, C. 2005, ApJ, 621, 695

van Bever, J., \& Vanbeveren, D. 1998, A\&A, 334, 21

Weidner, C., \& Kroupa, P. 2004, MNRAS, 348, 187

Weidner, C., \& Kroupa, P. 2005, ApJ, 625, 754

Weidner, C., \& Kroupa, P. 2006, MNRAS, 365, 1333

Weidner, C., Kroupa, P., \& Bonnell, I. A. D. 2010, MNRAS, 401, 275

Weidner, C., Kroupa, P., \& Larsen, S. S. 2004, MNRAS, 350, 1503

Weidner, C., Pflamm-Altenburg, J., \& Kroupa, P. 2011, in ASP Conf. Proc. 440,

UP2010: Have Observations Revealed a Variable Upper End of the Initial Mass Function?, ed. M. Treyer et al. (San Francisco, CA: ASP), 19

Weisz, D. R., Johnson, B. D., Johnson, L. C., et al. 2011, arXiv:1109.2905

Weisz, D. R., Skillman, E. D., Cannon, J. M., et al. 2008, ApJ, 689, 160

Werk, J. K., Putman, M. E., Meurer, G. R., et al. 2008, ApJ, 678, 888

Zhang, Q., \& Fall, S. M. 1999, ApJ, 527, L81 